OPEN ACCESS

Edited by:

Sandra Blaess,

University of Bonn, Germany

Reviewed by:

Gokul Kesavan,

Technische Universität Dresden,

Germany

Steffen Scholpp

University of Exeter, United Kingdom

${ }^{*}$ Correspondence:

Arne C. Lekven

alekven@bio.tamu.edu

Received: 02 June 2017

Accepted: 17 July 2017

Published: 03 August 2017

Citation:

Gibbs HC, Chang-Gonzalez A,

Hwang $W$, Yeh AT and Lekven AC (2017) Midbrain-Hindbrain Boundary Morphogenesis: At the Intersection of Wnt and Fgf Signaling.

Front. Neuroanat. 11:64. doi: 10.3389/fnana.2017.00064

\section{Midbrain-Hindbrain Boundary Morphogenesis: At the Intersection of Wnt and Fgf Signaling}

\author{
Holly C. Gibbs ${ }^{1}$, Ana Chang-Gonzalez ${ }^{1}$, Wonmuk Hwang ${ }^{1,2,3}$, Alvin T. Yeh ${ }^{1}$ \\ and Arne C. Lekven ${ }^{4 *}$
}

${ }^{1}$ Department of Biomedical Engineering, Texas A\&M University, College Station, TX. United States, ${ }^{2}$ Department of Materials Science and Engineering, Texas A\&M University, College Station, TX, United States, ${ }^{3}$ School of Computational Sciences, Korea Institute for Advanced Study, Seoul, South Korea, ${ }^{4}$ Department of Biology, Texas A\&M University, College Station, TX, United States

A constriction in the neural tube at the junction of the midbrain and hindbrain is a conserved feature of vertebrate embryos. The constriction is a defining feature of the midbrain-hindbrain boundary (MHB), a signaling center that patterns the adjacent midbrain and rostral hindbrain and forms at the junction of two gene expression domains in the early neural plate: an anterior otx2/wnt1 positive domain and a posterior gbx/fgf8 positive domain. otx2 and gbx genes encode mutually repressive transcription factors that create a lineage restriction boundary at their expression interface. Wnt and Fgf genes form a mutually dependent feedback system that maintains their expression domains on the otx 2 or gbx side of the boundary, respectively. Constriction morphogenesis occurs after these conserved gene expression domains are established and while their mutual interactions maintain their expression pattern; consequently, mutant studies in zebrafish have led to the suggestion that constriction morphogenesis should be considered a unique phase of MHB development. We analyzed MHB morphogenesis in fgf8 loss of function zebrafish embryos using a reporter driven by the conserved wht 1 enhancer to visualize anterior boundary cells. We found that fgf8 loss of function results in a re-activation of wht 1 reporter expression posterior to the boundary simultaneous with an inactivation of the wnt 1 reporter in the anterior boundary cells, and that these events correlate with relaxation of the boundary constriction. In consideration of other results that correlate the boundary constriction with Wnt and Fgf expression, we propose that the maintenance of an active Wnt-Fgf feedback loop is a key factor in driving the morphogenesis of the MHB constriction.

Keywords: MHB, mes/r1, Wnt, Fgf, constriction morphogenesis, two-photon fluorescence, image analysis, zebrafish

\section{INTRODUCTION}

The midbrain-hindbrain boundary (MHB), also called the isthmic organizer, has piqued the interest of developmental biologists for decades. Characterized by a conspicuous constriction in the developing neural tube, the MHB, located at the interface of the midbrain and hindbrain neuromeres, is well known to function as a signaling center responsible for patterning cell fates anteriorly in the midbrain and posteriorly in the cerebellum (Wurst and Bally-Cuif, 2001; Raible and Brand, 2004; Dworkin and Jane, 2013). The constriction is particularly evident in the dorsal neural tube and defines the posterior midbrain tectum and the hindbrain cerebellum. The MHB constriction also separates ventricular regions within the neural tube lumen, with the midbrain 
ventricle anterior to the constriction and the hindbrain ventricle behind. The MHB thus represents a crucial dividing point in the developing brain with characteristic morphological features which are critical for several MHB functions: as a signaling center, as a guide for neuronal migration and axon pathfinding (Volkmann et al., 2010), and as a physical separation of brain ventricles (Lowery et al., 2009). What is less well understood is the link between the mechanisms responsible for MHB specification and patterning, and between the signaling molecules that provide its signaling center activity and the constriction morphology that invariantly accompanies vertebrate $\mathrm{MHB}$ development. In other words, why is there always a neural tube constriction at the MHB, and is this morphology a cause, or consequence, of $\mathrm{MHB}$ function?

The mechanisms behind MHB specification and function are of interest on multiple levels. First, model organism studies have shown that defects in specification and patterning of the MHB lead to major deficiencies in the brain, such as the absence of midbrain, loss of cerebellum, and overgrowth of the midbrain tectum (McMahon and Bradley, 1990; Thomas and Capecchi, 1990; Buckles et al., 2004). Second, advances in magnetic resonance imaging (MRI) have enabled new analyses of human midbrain-hindbrain malformations (Doherty et al., 2013). These new imaging studies are revealing a surprising number of human central nervous system deficits that likely are caused by aberrant developmental patterning, such as the association of septo-optic dysplasia with chromosome 14 deletions, which include the neural patterning gene, ot $x 2$ (Severino et al., 2014). Identifying potential causes of these severe nervous system diseases requires a thorough understanding of the developmental mechanisms behind midbrain-hindbrain development.

As demonstrated by mouse mutants and zebrafish reporter lines, the $\mathrm{MHB}$ is specifically positioned within a domain of the early neural plate referred to as mes/r1 in mouse (Zervas et al., 2004) or the midbrain hindbrain domain (MH) in zebrafish (Tallafuss and Bally-Cuif, 2003). These studies show that the early mesencephalon (mes) and rhombomere 1 (r1) in the anterior hindbrain are genetically co-specified, and the MHB defines a balance point between these midbrain and hindbrain divisions. Besides positioning the future MHB, the balance point also represents an interface between Wnt ligand expressing progenitors of the posterior mesencephalon and Fgf ligand expressing progenitors of the anterior rhombencephalon, which interact in multiple ways throughout the specification and morphogenesis of mes/r1 and the MHB. Thus, an important question that is not yet sufficiently answered is what is the significance of the Wnt-Fgf interface at the MHB to mes/r1 development?

\section{MIDBRAIN HINDBRAIN DOMAIN MORPHOGENESIS AND PATTERNING}

To appreciate the difficulty of dissecting the role of Wnt and Fgf signaling families in the morphogenesis and patterning of the $\mathrm{MH}$ by the isthmic organizer, and to begin to identify processes whose disruption would result in neurological disorder, it is helpful to first have a clear picture of how the $\mathrm{MH}$ takes shape. A model of the current morphological and molecular ontogeny of the $\mathrm{MH}$ region in zebrafish is shown in Figure 1.

\section{Positioning}

The first critical step in $\mathrm{MH}$ morphogenesis is correct positioning of the neural primordium on the body axes such that spatiotemporal positioning cues can properly "posteriorize" the nervous system, that is, establish anteroposterior positional information after neural induction. Neural primordium positioning is mediated in part by the mechanical coupling of the presumptive neurectoderm to the involuted, anteriorlymigrating prechordal plate of the axial mesoderm via friction forces generated by the cell adhesion molecule E-cadherin, which is coupled on its cytoplasmic end to the canonical Wnt effector molecule, $\beta$-catenin (Smutny et al., 2017). In this study, uncoupled ectodermal cells anterior to the presumptive neurectoderm "flowed" laterally, posteriorly, and then medially. These complex "vortex" migrations of presumptive neurectodermal cells are presumably part of convergent extension movements that rely on non-canonical Wnt/PCP signaling to facilitate medial intercalation and anterior-posterior axis lengthening (Heisenberg et al., 2000; Davey and Moens, 2017). What is remarkable is that on their tumultuous journey, presumptive neurectodermal cells are precisely exposed to a complex molecular program that includes posteriorizing Wnt and Fgf signals emanating from the blastoderm margin (reviewed in Green et al., 2015; Tuazon and Mullins, 2015), resulting in a correctly patterned neural plate. One particularly remarkable and unknown aspect of this process is how early cell responses to Wnt and Fgf ligands occur during the complex morphogenetic changes of gastrulation.

Several lines of evidence suggest that Wnt and Fgf ligands expressed in the nascent paraxial mesoderm function as morphogens by establishing concentration or activity gradients that generate anteroposterior positional information that is interpreted into patterned cell fates (e.g., Cox and HemmatiBrivanlou, 1995; McGrew et al., 1995). A crucial function in particular for Wnt ligands in polarizing the neural plate has been established from experiments in zebrafish, Xenopus, and chick (Kiecker and Niehrs, 2001; Nordström et al., 2002; Dorsky et al., 2003; Rhinn et al., 2005). The mechanism by which graded Wnt activity is established is not yet known, though recent results suggest delivery of Wnt ligand via filopodia from paraxial mesoderm progenitors may be a major mode of transport (Stanganello et al., 2015). This differs from Fgf in this context, which has been shown to freely diffuse from its paraxial mesoderm source and form a gradient through a source-sink mechanism (Scholpp and Brand, 2004; Yu et al., 2009).

Of importance to the position of the MHB organizer, specifically, is the activation of the transcription factor $g b x 1$ in the posterior neural plate by Wnt8a (Rhinn et al., 2005, 2009), which antagonizes independently activated ot $x 2$ expressed in the forebrain and midbrain (Kurokawa et al., 2012). In the zebrafish, these domains overlap slightly at $60 \%$ epiboly but subsequently become mutually exclusive by $80 \%$ epiboly (Rhinn et al., 2003), 


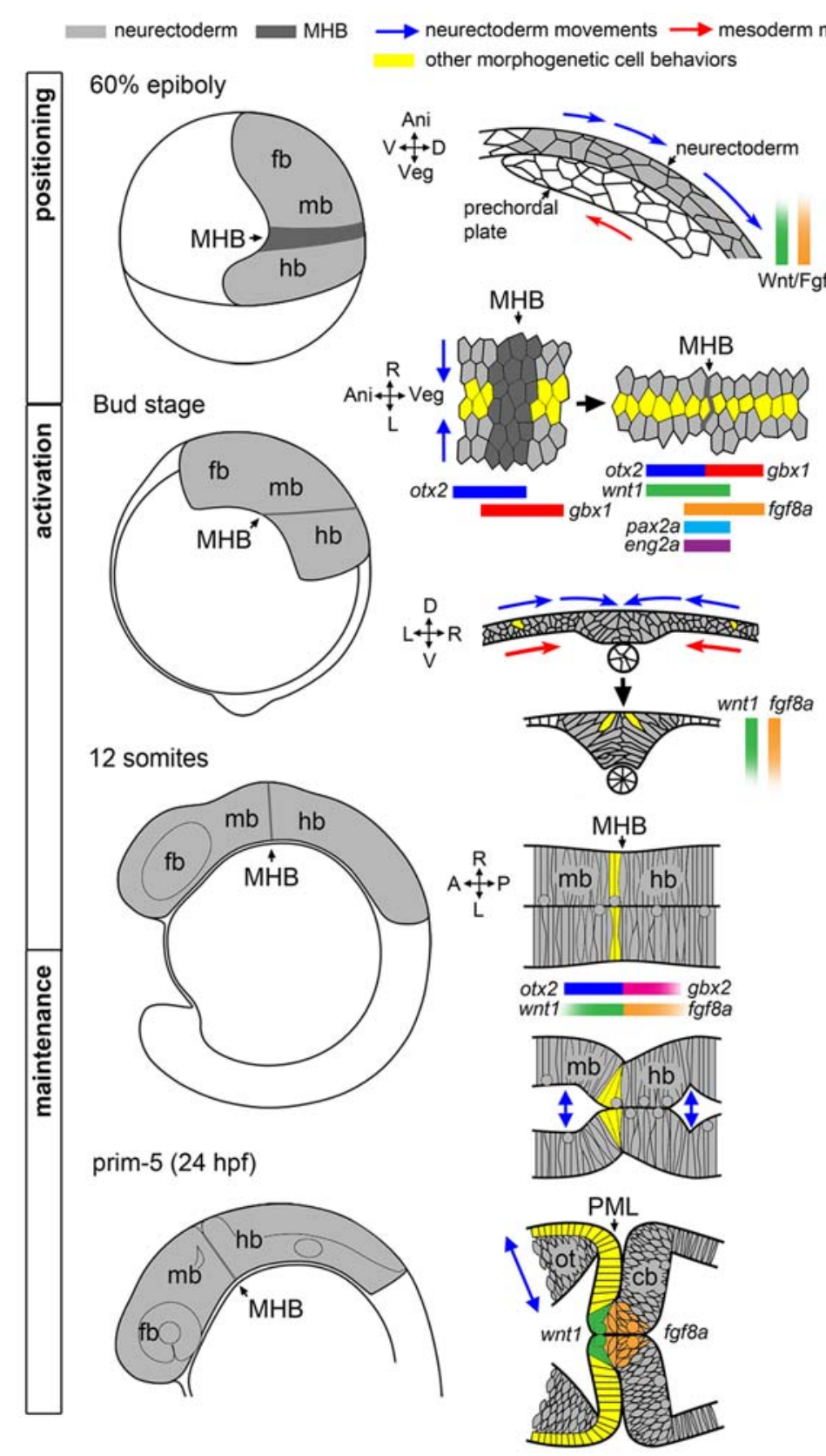

\section{Morphological forces and behaviors}

Friction forces between the prechordal plate and overlying neurectoderm (E-cadherin mediated)

Convergent extension movements and medial intercalation (PCP)

Lineage restriction

Mesoderm-directed

lateral-medial to

dorsal-ventral

internalization

Oriented cell divisions

on apical side of $\mathrm{nt}$

Neural crest migration

Ca++ signaling, Differential actomyosin contractility

Cell shortening, laminindependent basal constriction

Selective midline adhesion ventricle inflation

Peripheral midbrain layer (PML) formation

Cortical layering and epithelial pseudostratification

Neurogenesis and migration (PCP)
(Su 2013)

(Smutney 2017)

(Davey 2017)

(Langenberg 2005)

(Araya 2014)

(Lowery 2004)

(Tawk 2007)

(Klymkowsky 2010)

(Sahu 2017)

(Gutzman 2015)

(Filas 2012)

(Gutzman 2008)

(Recher 2013)

(Cooper 2015)

(Ninkovic 2005)

(Davey 2016)

FIGURE 1 | Morphogenetic and molecular ontogeny of the midbrain hindbrain boundary (MHB) in zebrafish embryos. Left column: schematic diagrams of zebrafish embryos, lateral view, at stages indicated on left. Gray shading highlights the brain primordium. Middle column: diagrams of pertinent morphogenetic movements. Orientation is indicated on the left side of each diagram. Examples of each morphogenetic property can be found in the corresponding references in the right column. fb, forebrain; mb, midbrain; hb, hindbrain.

while in mice there is initially a gap between Otx2 and Gbx2 that closes (Wurst and Bally-Cuif, 2001). In either case, there is a territory of cells that appears to be uncommitted to either the midbrain or hindbrain compartment that is consistent with observed variability in fate mapping experiments conducted before and after gastrulation (Woo and Fraser, 1995). However, strong evidence exists showing that by the time segmentation is underway in zebrafish (Langenberg and Brand, 2005), chick
(Tossell et al., 2011a,b), and mice (Sunmonu et al., 2011), the MHB is lineage restricted and cells in the midbrain and hindbrain compartments do not intermix, though this conclusion has not been without controversy (e.g., Jungbluth et al., 2001). Though proper compartmentalization is important for the later establishment of appropriately sized neural progenitor pools in the $\mathrm{MH}$, it remains an open question whether a physical or molecular mechanism precipitates lineage restriction at the 
MHB. A report from chick suggests there is a posterior shift in the position of the Otx/Gbx interface, such that it only coincides with the physical MHB constriction at later stages in development (Hidalgo-Sánchez et al., 2005). In zebrafish and in mice, however, the consensus is that the initial Otx/Gbx boundary definitively marks the future $\mathrm{MHB}$ constriction prior to when it becomes morphologically visible. Notch signaling, which classically causes cells to make such boundary decisions by amplification of small stochastic differences in gene expression followed by cell sorting, has been implicated in sorting Otx and Gbx cells at the MHB in chick (Tossell et al., 2011b). Reports from mice and medaka suggest other genetic interactions may refine the boundary, as Gbx2 interacts with Groucho repressors and can directly compete with POU transcriptional activators of Otx2 (Heimbucher et al., 2007; Inoue et al., 2012). Intercellular actinomyosin networks that have been shown to drive morphogenesis, such as during mesoderm invagination in Drosophila, may also play a physical role in lineage restriction at the $\mathrm{MH}$ (Kasza and Zallen, 2011) or possibly regional changes in cortical actin tension cause cells to sort to one side of the boundary or another (Heisenberg and Bellaïche, 2013).

\section{Activation}

Shortly after the positioning phase during gastrulation, a suite of MHB genes are activated in distinct domains around the ot $x 2 / g b x$ boundary as the neural plate undergoes neurulation to form the neural tube. Expression of wnt 1 anteriorly and $f g f 8 a$ posteriorly to the presumptive $\mathrm{MHB}$ (that is still not morphologically obvious) reinforce the ot $x 2 / g b x$ interface while her 5 , eng $2 a$, and pax $2 a$ are expressed on both sides of the boundary (Rhinn and Brand, 2001; Buckles et al., 2004). Which molecules and/or forces activate these core members of the more extensive MHB genetic program remains poorly characterized, and, surprisingly, this activation program can occur in the absence of at least parts of the positioning machinery ( $\mathrm{Su}$ et al., 2014). Once activated, the specific roles of each gene in promoting subsequent development within the $\mathrm{MH}$ (beyond providing spatial cues) is also not well understood, though several components of the MHB program appear to have roles in both fate specification and morphogenesis within the MH (Dworkin and Jane, 2013). For instance, her5 is known to inhibit neurogenesis during segmentation (Tallafuss and Bally-Cuif, 2003; Ninkovic et al., 2005) and to subsequently promote neural stem cell identity in adult zebrafish (Chapouton et al., 2006).

The establishment of the Wnt/Fgf signaling interface, however, is certainly crucial to the development of the $\mathrm{MH}$. Both Wnt1 ${ }^{-/-}$and $\mathrm{Fgf8}^{-/-}$mice fail to develop the entire $\mathrm{MH}$ region (McMahon and Bradley, 1990; Chi et al., 2003). In zebrafish, loss of several redundant Wnts (wnt3, wnt3a, wnt1 and $w n t 10 b$ ) recapitulates a similar phenotype (Lekven et al., 2003; Buckles et al., 2004) and the zebrafish fgf 8 a mutant ace lacks a cerebellum and MHB constriction, though the midbrain is present but unpolarized, resulting in aberrant retinotectal projections (Picker et al., 1999). Fgf8 has been deemed the most important "organizing molecule" based on results from implanting Fgf8-soaked beads at sites anterior and posterior to the MHB. In these experiments, Fgf8 was sufficient to induce tectal and cerebellar structures and an underlying Otx/Gbx boundary, while similar experiments for Wnt1 showed no significant re-patterning of the surrounding tissues (Martinez et al., 1999). Indeed, no gain of function analysis for all the other major MHB molecules in any organism has yielded such striking results. However, a study in which Otx2 and Fgf8 were simultaneously knocked down has challenged the idea that Fgf8 is required to pattern cell fates in the MHB. Foucher et al. (2006) showed that in the absence of Fgf8, if Otx2 levels were depleted, cerebellar neurons were able to successfully differentiate, though MHB morphology was abnormal in these embryos. Recent analysis of otx; $g b x ; f g f$ embryos also suggests that robust cerebellar differentiation requires Fgf (Su et al., 2014).

During MHB program activation, the process of primary neurulation, in which the neural plate coalesces on the dorsal midline, is ongoing (Lowery and Sive, 2004). During this process, the medial-lateral organization of the neural plate is transformed to a ventral-dorsal orientation (Schmitz et al., 1993). It is worth mentioning that although the subsequently developing $\mathrm{MHB}$ constriction has been studied primarily in reference to the $\mathrm{A} / \mathrm{P}$ axis, it is not uniform on the $\mathrm{D} / \mathrm{V}$ axis of the neural tube, which may reflect graded or inhomogeneous Wnt/Fgf activity along the $\mathrm{D} / \mathrm{V}$ axis and integration with dorsoventral patterning signaling activities (Lekven et al., 2003; Puelles et al., 2003). Thus, the MHB literature is largely focused on organizer activity in the alar region of the MH with relatively little known of the basal tegmentum.

Shortly after the neural tube is formed and the neural crest begins to migrate, the MHB constriction becomes a visible morphological feature as brain ventricles begin to form. In zebrafish, $\mathrm{MHB}$ constriction requires cell shortening and subsequent laminin-dependent basal constriction of a small ring of cells at the boundary (Gutzman et al., 2008). The cell shape changes involved in MHB constriction morphogenesis require non-muscle myosin II, and recent results show that cell shortening required at the $\mathrm{MHB}$ constriction is a consequence of calcium transient regulation of myosin light chain kinase (Gutzman and Sive, 2010; Gutzman et al., 2015; Sahu et al., 2017).

\section{Maintenance}

Once the neural tube is formed and the MHB constriction has been initiated, the genetic program within the $\mathrm{MH}$ subsequently transitions to the maintenance phase accompanied by continued reshaping of the brain tissue and ventricular system, as well as production of cerebrospinal fluid that may itself contribute to MHB regulation (Parada et al., 2005; Gato and Desmond, 2009). Computational modeling and experimentation in chick indicate importance of differential myosin-mediated contractility to produce brain ventricle geometry and suggest strategies may differ from compartment to compartment depending on the end fate of the junction, as some are only transient structures (rhombomere boundaries, for example) while others, such as the MHB constriction, persist as structures in the adult brain that must resist increasing fluid pressure from the ventricular system (Filas et al., 2012). One function the MHB constriction may play, thus, is as a point of transition between different anterior and posterior brain ventricle morphogenesis programs converging at the boundary. Such a structure would 
need to maintain cell adhesion at the boundary until brain ventricle morphology was established on either side to prevent misspecification of the surrounding tissues. The constriction could also mediate the timing of signaling between anterior and posterior brain compartments in the case of signaling molecules secreted in the cerebrospinal fluid. Such phenomena are not without precedent; for example, in mouse embryos it is well known that left/right asymmetry is broken by cilia- directed fluid flow in the node, though it is not known if the signal mediated through the unidirectional fluid flow is mechanical or chemical in nature (Yoshiba and Hamada, 2014). Brain ventricles have been shown to have cilia, and in zebrafish cilia in the developing telencephalon were shown to direct neuronal migration (Kishimoto et al., 2011). Some such mechanism may account for the evolution of the closed primary neurulation strategy seen in zebrafish compared to neural tube infolding seen in other vertebrates.

In the maintenance phase, several sub-regions of the $\mathrm{MH}$ emerge that execute their own morphogenetic programs in anticipation of neurogenesis. In the midbrain, the optic tectum is shaped by the formation of a tight sheet of cells called the peripheral midbrain layer (PML) harboring slow-cycling neural progenitor cells that will give rise to columns of neurons organized by alternating protocadherin expression that populate the more anterior tectum in a cortical fashion (Recher et al., 2013; Cooper et al., 2015; Rapaciolii et al., 2016). Posterior to the MHB, the cerebellar rhombic lip and ventricular zones form, from which granule and Purkinje progenitor cells are later derived, respectively, before their neural derivatives organize into the dorsoventral layers and mediolateral compartments that provide the foundation of the cerebellar circuitry (Hashimoto and Hibi, 2012; Millen et al., 2014). The MH tegmentum has almost no overt morphological landmarks apart from a relatively shallow constriction, but its correct patterning and morphogenesis is critical to the proper formation of serotonergic and cholinergic nuclei implicated in important behavioral functions (Parker et al., 2013).

The correct establishment and maintenance of each of the aforementioned pro-neural sub-regions of the $\mathrm{MH}$ requires at minimum a Wnt and Fgf signaling feedback loop to establish the proper unique molecular and mechanical microenvironments (Carletti and Rossi, 2008). In zebrafish ace(fgf $8 a$ ) mutants, the expression of wnt 1 and several other genes in the boundary region including her 5 , pax $2 a$, and eng $2 / 3$ are activated but their expression fades as Fgf-dependent feedback fails in early- to mid-somitogenesis (Reifers et al., 1998). Similarly, the combined loss of wnt1/wnt10b/wnt $3 a$ in zebrafish results in transient expression of $f g f 8 a$, pax $2 a$, and eng $2 / 3$ in the early $\mathrm{MHB}$ (Buckles et al., 2004). We have recently found using live multiphoton imaging (Gibbs et al., 2014a) that ace mutants form a constriction that fails to mature properly in the maintenance phase (Figure 2), a morphological transient output of a molecular transience (Gibbs et al., 2013). The failure of the constriction to continue morphogenesis in the maintenance phase is due to aberrant cell behaviors in two groups of cells. By imaging a transgenic wnt1 reporter line (Gibbs et al., 2014b) in the ace $(f g f 8 a)$ background, we identified one group of cells that fails to maintain wnt1 expression in the posterior midbrain, and to subsequently coordinate the proper morphogenesis of the PML and boundary tegmentum, and another group that fails to suppress wnt1 expression in the dorsal part of $\mathrm{r} 1$ to correctly specify the cerebellar plate (Figures 2, 3). This observation, based on identification of individual cells, supports previous reports of an isthmo/cerebellar-to-tectal transformation in molecular identity of the presumptive cerebellum that occurs with genetic reprogramming during the maintenance phase (Jászai et al., 2003; Gibbs, 2014), though with live imaging we observed that this reprogramming caused by a lack of $f g f 8 a$ does not preclude the previous initiation of the morphogenesis of the MHB during the activation phase. Thus, a mechanism independent of $f g f 8 a$ positions and initializes this physical boundary, while an $f g f 8 a$ dependent mechanism (either directly or indirectly) maintains its continued morphogenesis.

Wnt and Fgf signaling may also have more direct roles in shaping the $\mathrm{MH}$ during neurogenesis. As mentioned previously, during early stages of MHB formation, neurogenesis is actively inhibited by her 5 in zebrafish (Tallafuss and Bally-Cuif, 2003) but subsequently neurons are born as her 5 expression recedes to a narrow ring at the constriction. Wnt1 has recently been proposed to mediate the timing of neurogenesis in the midbrain by driving Fgf8 expression at the boundary and gradually suppressing it away from the boundary by inducing Sprouty expression so that Fgf dependent her5 also recedes (Dyer et al., 2014). Wnt1 may also function to promote neural stem cell identity in the dorsal midbrain and MHB (Miyake et al., 2012; Lin and Lee, 2016), possibly regulated by Fgf3/8-dependent Fgf22 signaling (Miyake and Itoh, 2013) and may contribute to shaping the $\mathrm{MH}$ by regulating the cytoskeleton during axon guidance (Ciani and Salinas, 2005). In the hindbrain, differentiation of unique tegmentum nuclei identities happens in spatiotemporal waves emanating from the upper rhombic lip. Recently, these migrations were shown to be conserved in mice and zebrafish, with discrete Wntl populations in the upper rhombic lip sequentially migrating anteriorly toward the MHB and turning ventrally to their final positions in the hindbrain tegmentum (Volkmann et al., 2010). Fgf9/Fgfr2 signaling is important for differentiation of Bergmann glial cells in the cerebellum of mice (Meier et al., 2014), a cell type conserved in the zebrafish cerebellum (although zebrafish do not appear to have an $f g f 9$, this function could be attributed to another redundantly functioning Fgf; Bae et al., 2009).

\section{MORPHOGENETIC ROLES FOR WNT AND FGF SIGNALING DURING CONSTRICTION FORMATION}

The Wnt and Fgf signaling pathways are expansive core developmental pathways that play a variety of contextdependent roles. In this section, we further examine the concept that a Wnt/Fgf signaling loop is required for proper $\mathrm{MH}$ morphogenesis and discuss potential points of crosstalk between these signaling pathways and cell adhesion and cytoskeletal machinery, based on studies both in the $\mathrm{MH}$ and other systems. 

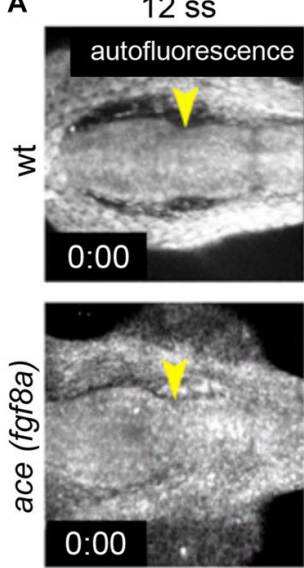
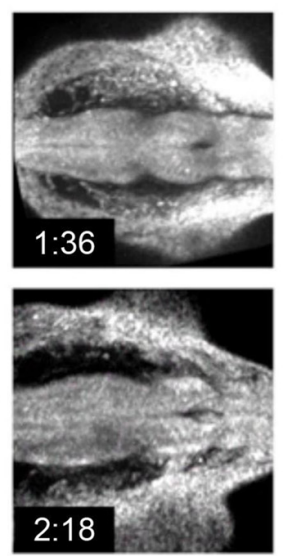
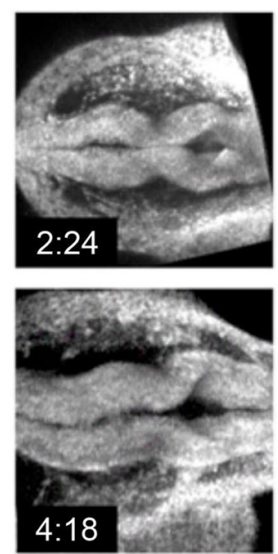

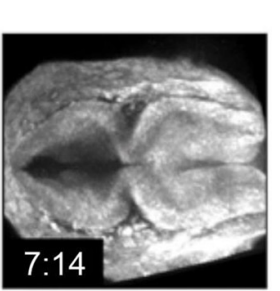

early pharyngula
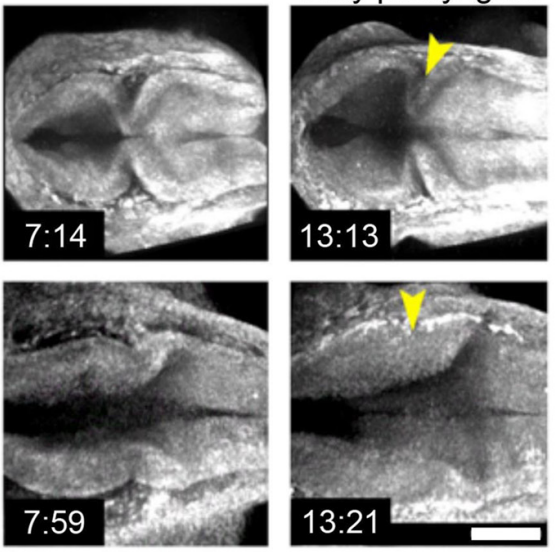

B
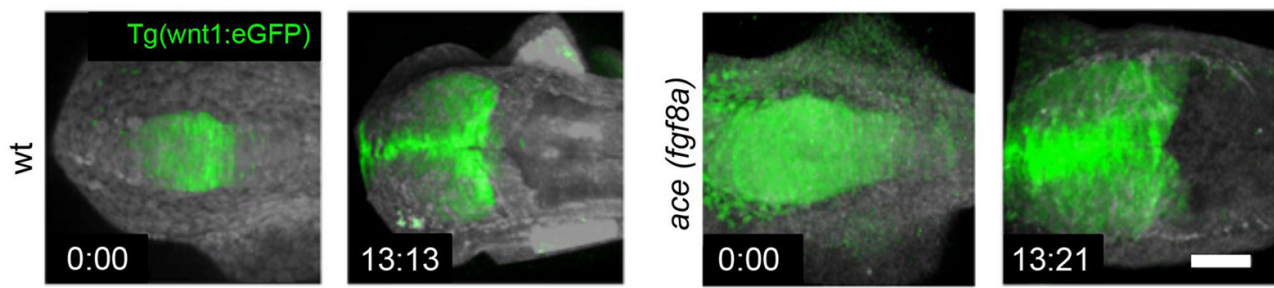

C
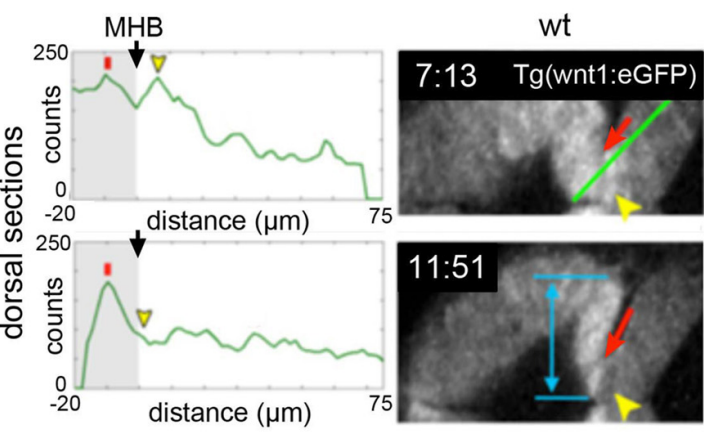

ace (fgf8a)
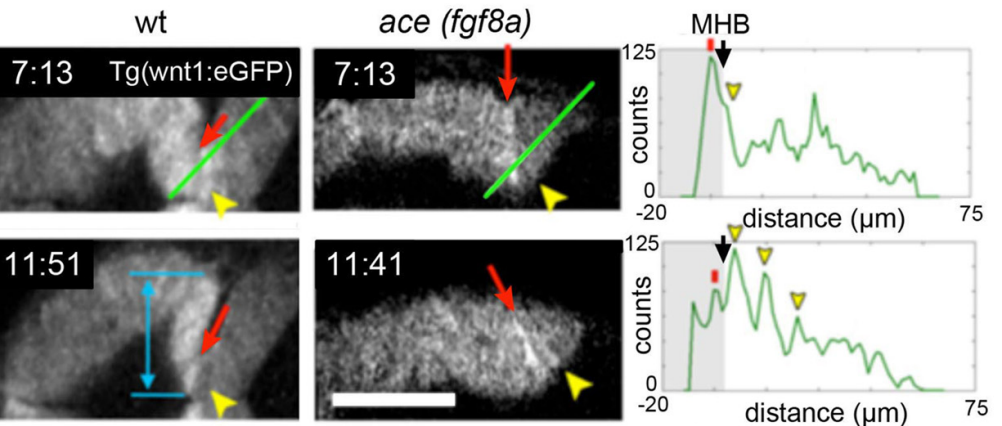

D

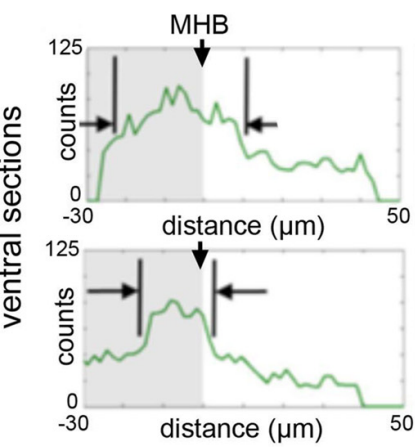

wt
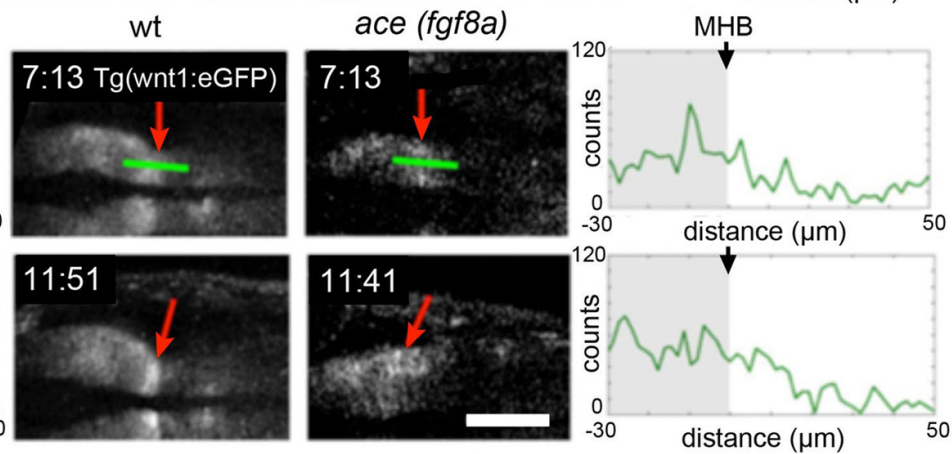

FIGURE 2 | Region dependent wht1 reporter response in ace(fgf8a) background. (A) 3-D reconstructions generated using maximum intensity projection from tissue autofluorescence (with sections from the roof plate removed) reveal the formation of an isthmic constriction (yellow arrowheads) that fails to mature without fgf8a. (B) wnt1 lineage is present but improperly polarized in ace embryos. During normal development, the wnt1 lineage increases expression of wnt 1 that can be visualized by increased eGFP reporter signal at the MHB boundary. (C) The wht1 lineage in the dorsal neuroepithelium normally turns off expression of wnt1 in the anterior hindbrain as reflected by a decrease in reporter intensity (measured from the profiles marked by green lines over time). Red arrows point to a midbrain cell just anterior to the MHB constriction and yellow arrowheads point to a neighboring cell posterior to the constriction. The blue arrow shows the presumptive peripheral midbrain layer (PML). In ace(fgf8a) embryos, the constriction relaxes and neighboring cells in $\mathrm{r} 1$ begin re-expressing wnt1 as shown by increasing reporter intensity, reflecting a cerebellar-to-tectal transformation. Basal constriction of the boundary cells with highest wnt1 reporter intensity occurs in both cases, however, the presumptive PML fails to form in ace(fgf8a) embryos. (D) The wnt1 lineage in the ventral neuroepithelium normally undergoes cell shortening and compresses to a narrow ring of cells anterior to the physical MHB constriction (black markers) while in ace(fgf8a) embryos, this reorganization fails to occur and wnt1 is no longer expressed. Scale bar $=100 \mu \mathrm{m}$. 

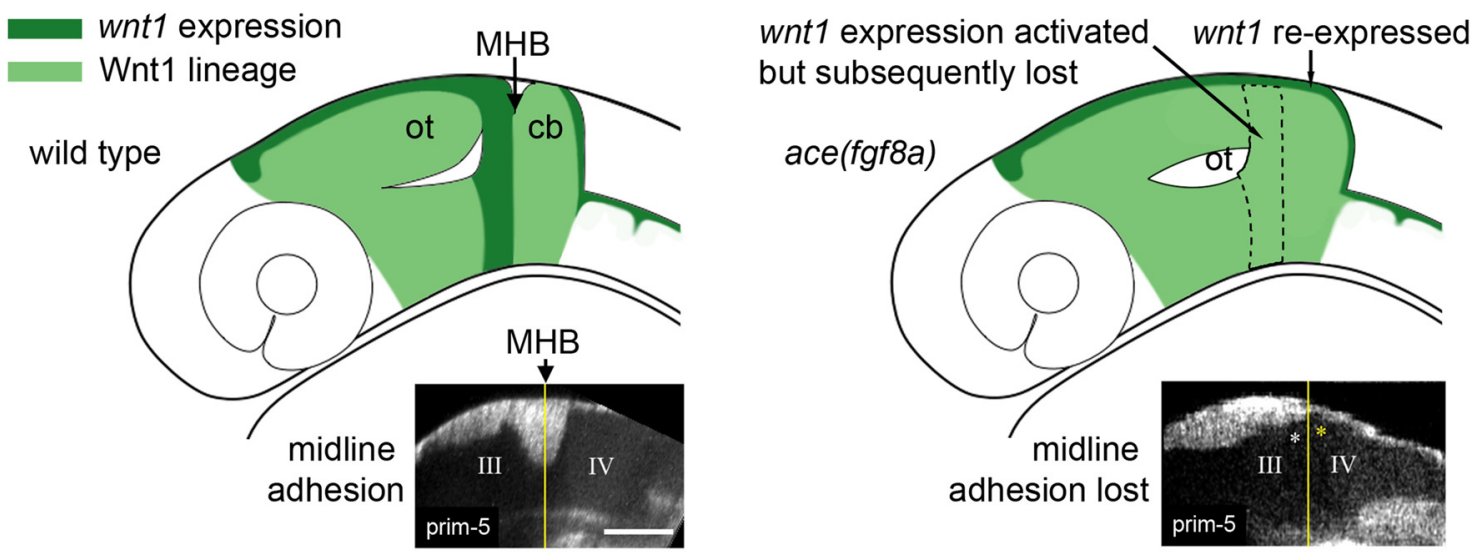

FIGURE 3 | Adhesion failure in ace(fgf8a) embryos and regionally dependent wnt1 lineage response. (Left) Initially broad expression of wnt1 is normally refined to the dorsal midbrain and anterior midbrain hindbrain domain $(\mathrm{MH})$, helping to maintain fgf8a dependent adhesion in the dorsal $\mathrm{MH}$. (Right) wnt1 expression in the posterior midbrain (except for a dorsal stripe) is lost in ace(fgf8a) while wnt1 expression in a dorsal stripe of $r 1$ is reactivated during a cerebellar-to-tectal transformation. Midline adhesion in the $\mathrm{MH}$ is not maintained during brain ventricle morphogenesis.

\section{Effect of Modulating Wnt/Fgf Signaling on MH Morphogenesis}

Loss of $f g f 8 a$ in the $\mathrm{MH}$ in ace mutants and subsequent modulation of wnt1 expression within the wnt1 lineage (Figures 2, 3) results in a failure of cells near the MHB constriction to carry out certain aspects of normal morphogenesis, including cell shortening, preserving midline adhesion, properly forming the brain ventricles, and organizing the presumptive PML (Gibbs, 2014). Yet, some mechanism (possibly Wnt-dependent) from the positioning or activation phase exists that initiates a transient constriction.

From live imaging, we can deduce that midline adhesion and constriction relaxation are decoupled from each other in the ace $(f g f 8 a)$ phenotype, as the time point at which the tracking began in Figure 2C was after adhesion was already lost in $\mathrm{r} 1$, yet the orientation of the cells at the boundary changed slowly over time from perpendicular to oblique (opposing the normal orientation) relative to the $\mathrm{A} / \mathrm{P}$ axis (Gibbs, 2014). We interpret these changes in orientation as reflecting another mechanism that contributes to the ace $(f g f 8 a)$ phenotype independent of adhesion loss. One possibility is a myosin-mediated epithelial relaxation step similar to the mechanism used to create transient constrictions between hindbrain rhombomeres (Gutzman and Sive, 2010), as non-muscle myosins have been shown to be important to MHB constriction formation (Gutzman et al., 2015). Without proper tension in the posterior midbrain epithelium, basal-constricting cells may fail to be mechanically stabilized by surrounding tissues, leading to a loss of proper boundary morphology. We have observed that the wnt 1 expressing cells in the dorsal posterior mesencephalon organize into a simple epithelium at the MHB that coincides with the presumptive PML. Perhaps this organization provides a local mechanical stiffness that allows basal constriction of boundary cells to result in a movement in the anterior vs. the posterior direction. Or, perhaps the basement membrane of that epithelium interacts with the basement membrane of $\mathrm{r} 1$ to stabilize the area during basal constriction.

We also tracked similar dynamics in the ventral region of the $\mathrm{MH}$ shown in Figure 2D, though signal attenuation at these imaging depths made it difficult to track individual cells (Gibbs, 2014). We observed that the wnt1 lineage initially spread across the boundary was subsequently compressed into a narrower region as the boundary angle sharpened (black markers). It was not clear if this behavior was a result of basal constriction. In contrast, in ace (fgf8a) embryos, the wnt1 lineage initially spanning the boundary failed to shorten, compress, and organize into a ring of cells, though their orientation did seem to transition from perpendicular to oblique, perhaps as a result of forces resulting from constriction failure in the dorsal neuroepithelium. From this analysis, it would seem there are different mechanical forces exerted on the isthmic constriction along the dorsoventral axis of the tube during somitogenesis, which would potentially lead to different strategies for maintaining the mechanical integrity of the boundary for proper formation of the surrounding tissue.

Previously, increased levels of cell death were observed in dorsal $\mathrm{r} 1$ in ace mutants during mid-somitogenesis (Reifers et al., 1998) and attributed to apoptosis following an r1-tomesencephalic identity change. A similar $\mathrm{Fgf8}^{-/-}$phenotype was observed in mice (Chi et al., 2003). While such a phenomenon could potentially explain the loss of adhesion we have seen in dorsal $\mathrm{r} 1$, we have not observed any increase in autofluorescence signals that may indicate such events occurring in ace $(f g f 8 a)$ embryos. Instead, we argue that $f g f 8 a$ plays a role in maintaining adhesion during normal development. We also observed in ace $(f g f 8 a)$ embryos that hindbrain opening initiates normally, but spreads anteriorly into the $\mathrm{MH}$, possibly due to the failure to maintain the MHB genetic program without $f g f 8 a$ (Gibbs, 2014). Insufficient Fgf signaling may thus render the dorsal $\mathrm{MH}$ competent to a signal emanating from the $\mathrm{r} 1 / \mathrm{r} 2$ boundary 

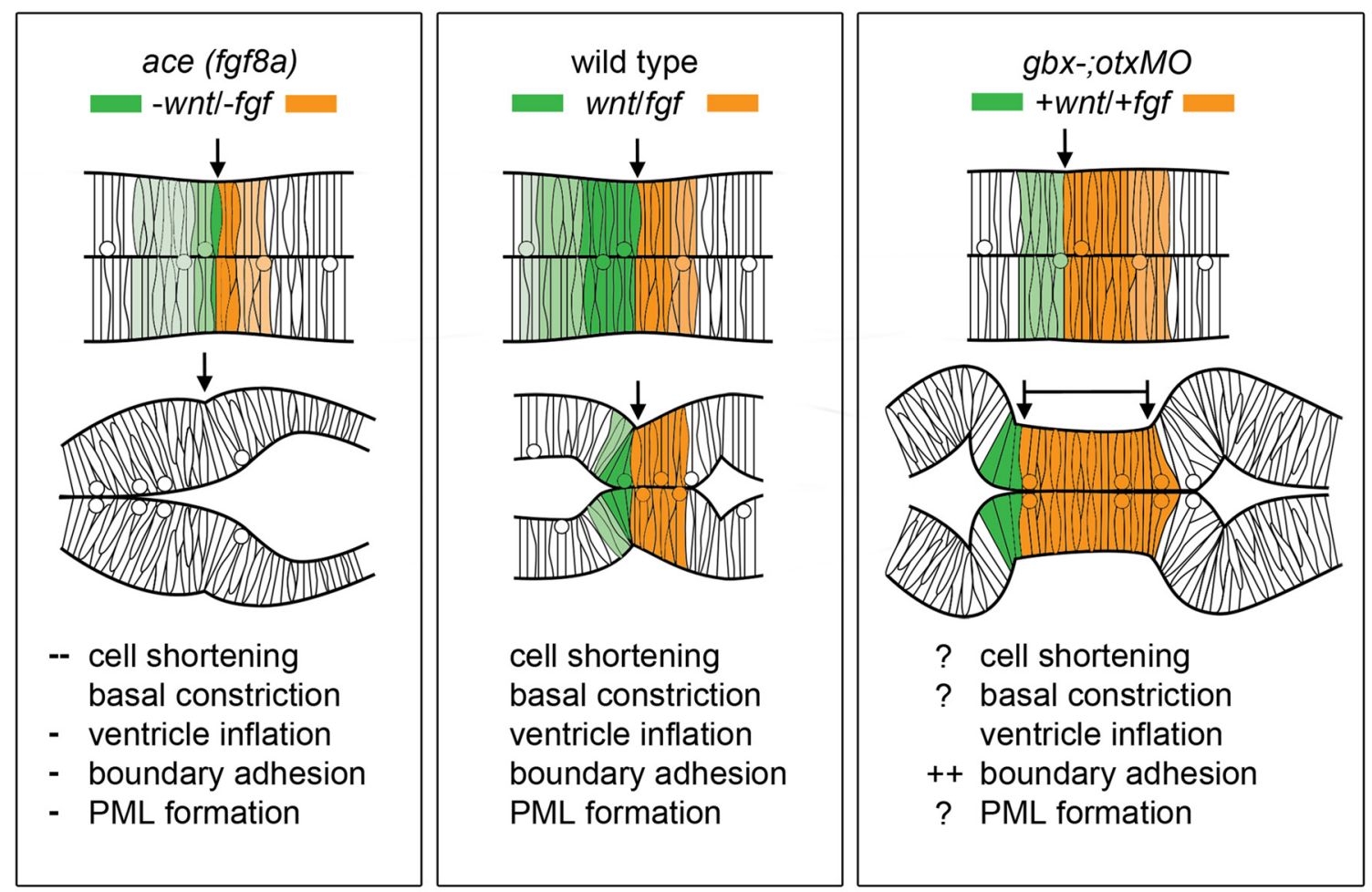

FIGURE 4 | Modulation of Wnt/Fgf signaling and effect on MH morphology. (Left) Summary of wnt/fgf expression domains, MH morphology, and morphogenetic cell behaviors in ace mutants. (Middle, Right) The same model shown for wild-type embryos and otx/gbx loss of function embryos. Figure drawn based on data found in Su et al. (2014). Deficient cell behaviors are indicated with a minus sign (-), while overactive cell behaviors are indicated with a plus sign (+).

that triggers hindbrain ventricle opening (Gutzman and Sive, 2010). Interestingly, the limits to which the initially broad fgf $8 a$ domain narrow (initially spanning r1-r4 and narrowed to discrete domains in $\mathrm{r} 1$, ventral $\mathrm{r} 2$, and $\mathrm{r} 4$; Reifers et al., 1998), mirrors the curious sequence of hindbrain ventricle opening in more posterior rhombomeres. In zebrafish, ventricles open first at the dorsal $\mathrm{r} 1 / \mathrm{r} 2$ interface (Gibbs, 2014), followed by small openings at the r3/r4 and r4/5 boundaries (Gutzman and Sive, 2010). The timing of $\mathrm{r} 4$ separation reported by Gutzman and Sive (2010) occurs at seemingly the same time $f g f 8 a$ expression is lost in r4 (Reim and Brand, 2002). As forebrain domains of $f g f 8 a$ expression also correspond with areas that shape forebrain ventricle morphology, and a large $f g f 8 a$ domain at the boundary of otx/gbx loss of function embryos corresponded with an abnormally long constriction where cells seemed unable to properly delaminate at their apical interface (Su et al., 2014), it is reasonable to hypothesize that $f g f 8 a$ may be a necessary factor to maintain adhesion at brain ventricle boundaries.

Figure 4 summarizes the effects of modulating Wnt/Fgf signaling on morphogenic cell behaviors in the $\mathrm{MH}$ during the maintenance phase of formation. Complete knock down of $f g f 8 a$ and transient, low levels of wnt 1 expression in ace embryos lead to an opposite adhesion phenotype than that seen when $f g f 8 a$ expression is initiated in an unrestricted manner throughout the $\mathrm{MH}$ and subsequently activates late $w n t 1$ signaling in $o t x / g b x$ loss of function embryos (Su et al., 2014). The role played by $f g f 8 a$ in maintaining adhesion is likely indirect. Adhesion loss at the midline progresses strikingly in step with the loss of eng2 and pax2a expression patterns in ace at the MHB (Reifers et al., 1998) and eng2, like $f g f 8 a$, is not present in $\mathrm{r} 4$ when ventricle IV opens there. It would be interesting to examine these patterns in detail to see if eng 2 or pax $2 a$ are expressed more in cells at the midline and potentially mediate adhesion maintenance. wnt1, eng1.b, pax $2 a$, and il17rd overlap with $f g f 8 a$ in that region and would be candidate genes that help to mediate this $f g f 8 a$ dependent separation in the $\mathrm{MH}$. The timing of wnt1 expression, whether occurring only during the activation phase (as in ace $(f g f 8 a)$ ) or during the maintenance phase (as in $o t x / g b x$ loss of function), may be more important to cell behaviors such as constriction initiation, cell shortening, and PML formation in addition to restricting $f g f 8 a$ anteriorly.

\section{Wnt/Fgf Crosstalk with Cell Adhesion and Cytoskeletal Machinery}

Throughout the positioning, activation and maintenance phases of $\mathrm{MH}$ formation, it is established that canonical and non-canonical Wnt signaling activity, Fgf signaling activity, as well as precise modulation of cell adhesion, polarity, and motility, is required for correct shaping of the germ layers, the neural primordium, and subsequently the $\mathrm{MH}$. These molecular interactions that may instruct the cell behaviors leading to 


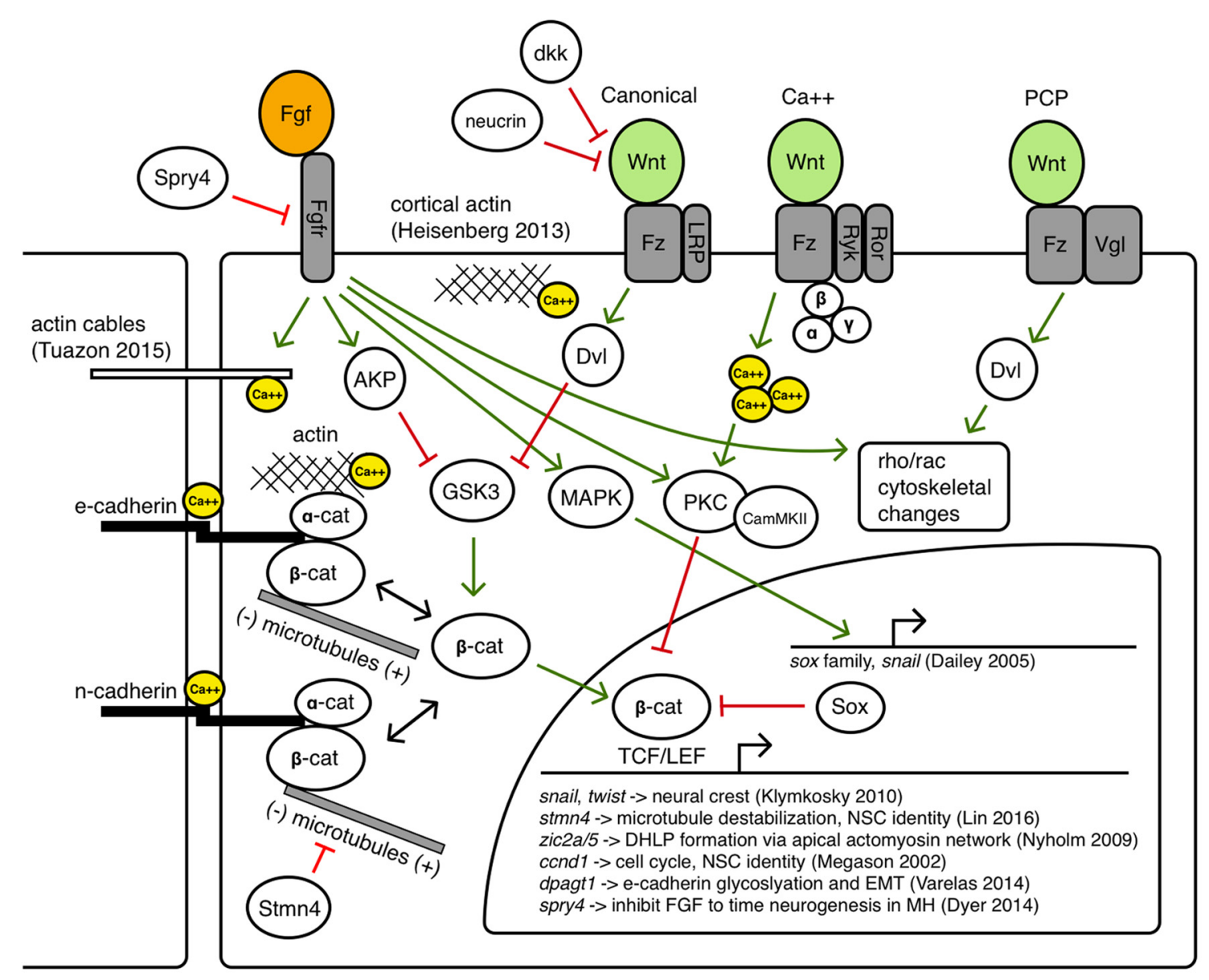

FIGURE 5 | Potential avenues of Wnt/Fgf signaling contributing to MH formation.

these morphogenetic changes are summarized in Figure 5. Within the $\mathrm{MH}$, there is likely to be spatiotemporally varying competencies to these interactions, but as they have not been precisely determined in this particular biological system, they are shown together in a single cell as possible avenues for further investigation.

Canonical Wnt signaling has long been associated with the build-up of a pool of cytoplasmic beta-catenin by inhibiting its degradation so beta-catenin can translocate to the nucleus and regulate target genes with TCF/LEF binding sites (Moon et al., 2004). However, both Wnt and Fgf signaling can inhibit GSK3, the primary component of the destruction complex that modulates beta-catenin levels (Dailey et al., 2005). Thus, a careful balance of Wnt/Fgf signaling may affect Wnt target genes during $\mathrm{MH}$ development such as snail (Yook et al., 2005) and twist (Klymkowsky et al., 2010), zic2a/5 (Nyholm et al., 2009), cyclinD (Megason and McMahon, 2002), stmn4 (Lin and Lee, 2016), and dpagt1 (Varelas et al., 2014), genes known to affect cell proliferation, dorsolateral hinge-point (DLHP) formation, and adhesion. Classical Fgf signaling may also be important in Bergman glial fate specification in the cerebellum (Meier et al., 2014). Fgf/MAPK signaling can also activate Sox proteins that may inhibit beta-catenin/TCF/LEF interactions, as well as activate Snail, which promotes epithelial-mesenchymal transition (EMT) by inhibiting cadherins (Dailey et al., 2005).

Crosstalk between Wnt signaling and beta-catenin mediated cell adhesion via binding with cadherins has been an area of ongoing research, revealing a large number of context-dependent points of interaction in which Wnt signaling can modulate cell adhesion and vice versa through the shuttling of beta-catenin between a cytoplasmic pool that can become a nuclear effector and a membrane pool that interacts with the cytoskeleton (Heuberger and Birchmeier, 2010). How these interactions may help shape the MHB constriction and other regions in the $\mathrm{MH}$ is not known. Zebrafish $\mathrm{N}$-cadherin (cdh2) is expressed throughout the nervous system and the $c d h 2$ mutant parachute has significant loss of midline adhesion in the neural tube (Lele et al., 2002). The down-regulation of $c d h 2$ is accomplished in migrating neural crest cells in a Wnt-dependent manner (Piloto and Schilling, 2010). Zebrafish E-cadherin (cdh1) is not expressed in the neural tube until sometime after the 16 somite stage, and is expressed in the presumptive MHB by $24 \mathrm{hpf}$ in a region appearing to overlap with Wnt expression near the midline (Babb et al., 2001). E-cadherin may be uniquely 
responsible for maintaining cell adhesion to help stabilize cytoskeletal rearrangements at the midline in the $\mathrm{MH}$ region or during the formation of the PML. One example linking Fgf signaling with morphogenetic remodeling of the cytoskeleton has been proposed to work through Fgfr-Ras-MAPK signaling in the formation of the lateral line sensory system in zebrafish (Harding and Nechiporuk, 2012). In this study, authors found that Ras-MAPK signaling activated by Fgfr was required for the formation of rosettes by localizing Rho-associated kinase (Rock) to the apical surface to drive its constriction. Fgf signaling has also been shown to have a role in otic vesicle formation, which requires apical constriction mediated by local increases in actin. In the otic vesicle, Fgf signaling activates phospholipase-C (PLC) which triggers non-canonical myosin-II activity (Sai and Ladher, 2008). Classically, myosin-II is understood to ratchet along actin filaments to promote contraction, however, upon phosphorylation by PLC, myosin-II promoted the degradation of basal actin (resulting in enriched apical actin and otic cup invagination). Wnt signaling has also been implicated in cytoskeletal remodeling via the planar-cellpolarity pathway and perhaps also canonical signaling pathways (Lapebie et al., 2011). Once neurogenesis begins in the $\mathrm{MH}$ region, it is possible that Wnt/Fgf signaling may modulate neural migration (Knosp et al., 2015). Wnt and Fgf have been implicated in changes in epithelial cell adhesion in neurogenic cranial placodes (Lassiter et al., 2014) and it has been shown that novel Wnt receptors Ryk and Ror can interact with the cytoskeleton to promote axon guidance (Clark et al., 2012).

Balance of intra- and intercellular calcium is another interesting candidate target bridging Wnt/Fgf signaling with cell adhesion and cytoskeletal dynamics (Kim et al., 2011; Tsai et al., 2015). Fgf signaling can promote intracellular calcium release and affect cytoskeletal organization through calcium and calmodulin dependent protein kinases (Schlessinger, 2000), a function that can also be accomplished by Wnt (Babb et al., 2001; Cohen et al., 2008) and may be combinatorial in the formation of the MHB constriction. The findings of Gutzman et al. of calcium transients that appear to drive myosin-dependent cell shortening in the posterior midbrain highlight the potential of such a morphogenetic role for Wnt/Fgf in the MH (Gutzman et al., 2015; Sahu et al., 2017). Additionally, how extrinsic and intrinsic physical forces triggering mechanotransduction pathways may intersect with tissue patterning pathways continues to be an active area of research (Heisenberg and Bellaïche, 2013).

As Figure 4 summarizes, several cell behaviors in the $\mathrm{MH}$ region during the maintenance phase appear to depend on Wnt/Fgf signaling, but studies are just beginning to identify which molecular components of the cell adhesion, cytoskeletal, or mechanotransduction machinery are responsible for particular behaviors and have not yet enumerated the direct pathways downstream of Wnt or Fgf that act in this region. Loss of Fgf8a signaling and resulting failure to maintain wnt1 expression in ace $(f g f 8 a)$ mutants result in the failure of cells to properly shorten at the constriction, aberrant ventricle inflation and morphology in the posterior midbrain, loss of midline adhesion at the constriction, and the failure of the PML to begin to form; yet, basal constriction of the boundary cells does occur.
Expanded Wnt/Fgf signaling (with Wnt signaling not becoming active until the maintenance phase) in $g b \times 1 / 2$ mutants with otx 2 knockdown results in a large constriction due to excessive midline adherence, apparently normal ventricle inflation and unknown effect on cell shortening, basal constriction, and PML formation. Figure 5 depicts potential avenues connecting Wnt/Fgf signaling and possible downstream effectors of these cell behaviors that continue to require further investigation as it remains difficult to study or visualize how multiple signaling inputs are transduced by secondary molecules and downstream effectors to regulate gene transcription, cytoskeletal dynamics, cell adhesion, and subsequent cell behavior in vivo.

\section{NEW TOOLS FOR ADDRESSING AN OLD MODEL ORGANIZER}

Despite all we know about Wnt and Fgf signaling and MHB development, significant questions, such as how tissue patterning via cell signaling intersects with the generation of morphogenetic force and cell shape change, remain unanswered. One roadblock to significant further progress has been the lack of appropriate enabling technologies to visualize both cell identity and cytoskeletal changes. Classically, morphogenesis and patterning have been studied in a relatively disconnected manner due to technological limits regarding the scale and precision of genetic and embryological manipulations and molecular labels, the spatiotemporal and spectral resolution of imaging systems, difficulty automating sophisticated image processing tasks, and minimal collaboration among developmental biologists, physicists, and engineers. These limitations are all manifestly highlighted by the gaps in understanding regarding the mechanistic patterning and morphogenesis of the $\mathrm{MH}$ region. In this section, we briefly discuss recent advances in imaging and image processing technologies we hope will help enable the assembly of a more spatiotemporally comprehensive model of $\mathrm{MH}$ patterning and morphogenesis.

\section{Imaging}

The images presented in Figure 2 were acquired with a homebuilt, ultrashort pulse microscopy (UPM) system configured to render intensity images using two-photon excited fluorescence. In our UPM system, sub-10-femtosecond pulses from a passively mode-locked Ti:Sapphire oscillator are coupled by a dualaxis, galvanometer-driven scanner into an upright microscope. The zebrafish embryos were mounted in agarose wells and submerged for coupling with water immersion objectives. The upright geometry is advantageous for manually aligning the region of interest in the embryo with the optical axis of the microscope. Generated two-photon excited fluorescence is collected in back-reflected geometry by the microscope objective and directed to photon-counting photomultiplier tubes for image rendering. In this configuration, our UPM system is pointscanning wherein images are rendered digitally pixel-by-pixel (Gibbs et al., 2014b).

The experimental configuration of our point-scanning UPM system is similar conceptually to laser scanning confocal 
microscopy (LSCM) systems, and comparisons between LSCM and two-photon laser scanning fluorescence microscopy (2PM) have been well discussed (Gao et al., 2012). Here, we emphasize two points, those of signal generation and photobleaching. Fluorescence signal used in LSCM is generated following the linear absorption of incident photons; linear absorption may be verified by a linear relationship between incident laser intensity and fluorescence signal. Indeed, "one-photon" fluorescence is a readily observable phenomenon such that signal is generated throughout the excitation beam path within the sample. Thus in LSCM, a pinhole confocal with the object plane is placed in front of the detector to discriminate against out-of-focus signal so that thin optical images may be rendered.

Fluorescence signal used in $2 \mathrm{PM}$ is generated following the nonlinear absorption of incident photons, i.e., simultaneous absorption of two photons. Two-photon absorption may be verified by a quadratic relationship between incident laser intensity and fluorescence signal. This nonlinear relationship between incident laser intensity and fluorescence manifests in limiting signal generation to the focus of the beam. Thus in $2 \mathrm{PM}$, fluorescence detection may be optimized for collection because the signal (point) source is assumed to be the focus of the incident beam. This optimization of collection combined with near-infrared excitation leads to a general advantage of 2PM over LSCM to acquire images from greater depths within thick, biological samples (e.g., the MHB throughout the entire DV axis).

Every absorptive event, whether linear or nonlinear, is potentially catastrophic to the emission properties of the fluorophore. Photobleaching is facilitated by the absorbed energy through which photo-induced damage, chemical modification, and environmental factors contribute to fluorophore fading. Since photon energy absorption is essential to the fluorescence process, the potential for photobleaching is unavoidable. In this respect, $2 \mathrm{PM}$ is more frugal than LSCM in its use of fluorophores, which can be an important consideration in live cell imaging studies over developmental time periods (e.g., to visualize the development of the MHB lineage restriction boundary).

Recent advances in live cell imaging have led to the development of systems that can comprehensively capture morphogenetic movements and divisions over multiple developmental stages. These recent advances in "in toto" imaging have been aided by the capability to sensitize high resolution microscopy techniques to single nuclei using genetically-encoded fluorescent markers such as fusion of histone and green fluorescent protein, though endogenous signals have been used to image and track every cell in the zebrafish embryo to create a lineage tree through its first 9 cell divisions (Olivier et al., 2010). Localized to nuclei, fluorescent markers of adjacent cells are well separated which is important for their delineation within the crowded environment of the embryo. Lineage tracing in this context then becomes an exercise in tracking progenitors and their progeny, albeit a challenging informatics exercise, especially in tissues with high cell density such as the forming MHB. This challenge has driven advances in imaging technology and computer aided analyses (Peng, 2008) that have revealed, with high spatial and temporal resolution, collective cell migrations (McMahon et al., 2008) and even divisions and movements of every cell within a developing zebrafish embryo over a $24 \mathrm{~h}$ period (Keller et al., 2008).

Light sheet microscopy, which has been developed utilizing fluorescence from linear and nonlinear absorption, has recently emerged in the developmental biology community (Keller et al., 2010; Santi, 2011; Weber and Huisken, 2011; Huisken, 2012) and goes by several names including SPIM (selective plane illumination microscopy), mSPIM (m for multidirectional), and DSLM (digital scanned laser light sheet microscopy), denoting differences in configuration and formation of the light sheet (Keller and Stelzer, 2008). The basic principle of SPIM was developed in 1903 by Siedentopf and Zsigmondy (Huisken, 2012), but as with LSCM, the technique did not impact the biological community until much later when in 2004, SPIM was used in vivo to image both the relatively transparent medaka embryo and more opaque Drosophila embryo (Huisken et al., 2004). To create a sheet of light, Huisken et al. (2004) used a cylindrical lens, which focuses light along one axis instead of two, as a spherical lens does, creating a sheet of light rather than a line. The sheet is scanned through the sample and the signal is detected by an objective lens placed at a $90^{\circ}$ angle that images onto a charged-coupled device (CCD) array. This parallelized excitation and detection renders an entire image onto the CCD camera, greatly increasing acquisition speed especially when compared with point-scanning microscopies.

More recently, light sheets have been created by fast-scanning a laser beam with a long depth of field or confocal parameter along one axis (DSLM), providing significantly higher signal to noise ratios than previous approaches (Keller and Stelzer, 2008). In demonstrating DSLM, Keller et al. (2008) characterized nuclear movements in zebrafish over the first $24 \mathrm{~h}$ of development for both wild type and Mzoep mutant embryos. Keller et al. (2008) found that the mechanism of hypoblast formation during epiboly varied by position, with dorsal mesendoderm forming by ingression and ventral mesendoderm by involution. Mzoep mutants failed to internalize cells to form the hypoblast.

In the light sheet configuration, the excitation beam path is in the object plane of detection and, thus, generation of fluorescence signal used to render images is confined to the plane of imaging. Therefore, whether fluorescence is generated by linear or nonlinear absorption, excited fluorophores contribute signal to image rendering before its unavoidable loss to photobleaching. This economical use of fluorophores compares favorably with LSCM that generates signal widely and then discriminates against out of focus fluorescence. In optimizing light sheet microscopy for live cell imaging and minimizing photobleaching in particular, different configurations have been developed to maximize acquisition speed and resolution while homogenizing illumination and minimizing peak intensities, e.g., lattice light sheet microscopy (Chen et al., 2014).

It is now possible to image with high (sub-cellular) resolution and to track the divisions and morphogenetic movements of every cell within a developing embryo over multiple developmental stages. With these technological developments, and with developments in genetic and embryological manipulations to generate genetically encoded 
molecular labels, constituent specific imaging and characterizing the interactions of multiple constituents in the developing embryo are now possible and may enable multicomponent analysis of complex biological systems. With fluorescence-based microscopy techniques, multiconstituent imaging will require different labels, excitation of those labels, their detection and image segmentation.

Technical hurdles to multiconstituent imaging exist but are rapidly being addressed. One issue is excitation and detection of multiple fluorophores in a single sample. The emission maximum of fluorescence from linear absorption is lower in energy than the absorbed photon and, therefore, spectrally shifted from its excitation wavelength. The detection of fluorescence signal may thus be achieved with the spectral rejection of the excitation laser from the detection path. With multiple fluorophores, it may be possible to tune the excitation to within overlapping absorption spectra of the fluorophores, tune an excitation laser for each fluorophore, or some combination thereof. However, with multiple fluorophores, one or more excitation laser wavelengths may overlap with fluorescence signal. Therefore, in spectrally rejecting the excitation lasers, some signal may be rejected as well. One approach to avoid rejection of signal is to sequentially excite the fluorophores, which also aids in image segmentation, albeit at the cost of image acquisition speed (Valm et al., 2017).

The emission maximum of two-photon excited fluorescence is higher in energy than the absorbed photons. In fact, the excitation wavelength is usually far removed spectrally from the detection window, i.e., near-infrared excitation wavelengths for fluorescence in the visible region. Thus, to excite multiple fluorophores, the central wavelength of the excitation laser pulses may be tuned to within overlapping nonlinear absorption spectra of the fluorophores, one may tune an excitation laser oscillator for each fluorophore (though not cost effective), or some combination thereof. Nevertheless, simultaneous excitation and detection of multiple fluorophores is achievable without any loss of signal from rejecting the excitation laser pulses. One approach is to use an ultrashort laser pulse that has a broad excitation spectrum to nonlinearly excite multiple fluorophores simultaneously (Gibbs et al., 2014a). Segmentation of the images may be achieved through spectral unmixing. This approach has an added advantage of image co-registration because a single laser is used to acquire the multicomponent images. Other schemes have, for example, elegantly used nonlinear optics to generate multicolor images of neural circuit formation in combination with the "brainbow" labeling system (Mahou et al., 2012).

Rapid advances in imaging technologies coupled with new methods for chromosome engineering via CRISPR/Cas9 (for example, see Sander and Joung, 2014) promise to usher in a new period of rapid advances based on these technologies to understand MHB development. For instance, the ability to engineer specific genomic loci to express fluorescent reporters of developmentally important genes will allow a greater understanding of cell lineage behaviors in imaging-friendly organisms. As one example, Ota et al. (2016) used CRISPR/Cas targeting to generate an eGFP expressing allele of zebrafish pax $2 a$
(Ota et al., 2016). By combining lineage and cytoskeletal reporters in multiconstituent imaging experiments, one could monitor the contributions of specific cell types to MHB morphogenesis. Alternatively, new high resolution labeling and imaging methods could enable the visualization of specific chromosomal loci and enhancers within defined cell lineages to understand the genomic control of cell fates within the MHB. These new tools are rapidly expanding our ability to visualize aspects of MHB development that were previously obscured from view.

\section{Image Analysis}

Another current limitation to characterizing dynamic changes in the developing MHB is the state of image analysis software. The number and breadth of image analysis tools are increasing. Use of these tools ranges from general application programs such as ImageJ and Icy (de Chaumont et al., 2012; Schindelin et al., 2015) to application-specific tools (Peng, 2008; Eliceiri et al., 2012). Development of these tools is driven by the need to rapidly and quantitatively analyze large, multi-modal image datasets. Such data vary in image types, time-series of $2 \mathrm{D}$ or $3 \mathrm{D}$ images, and multi-channel measurements. There are also many aspects of image analysis, such as visualizing pixel data in more informative ways, making automated measurements when images have distinct features or landmarks, and building structural models of the system being imaged. Despite the plethora of available software, visualizing zebrafish morphogenesis, especially during early stages of development, poses a considerable challenge since distinct landmarks (Mikut et al., 2013) may have not developed yet in these systems. Currently, there is no single software that can process an image stack and render a $3 \mathrm{D}$ reconstruction model of the imaging volume. Remarkably, even though the early-stage zebrafish embryo is relatively featureless (landmarkfree), humans can easily recognize its shape. The fact that human brains can process the image and construct a mental map of the zebrafish embryo suggests that it is in principle possible to computationally generate a similar 3D model. A 3D model refers to surfaces and anatomical volumes "recognized" or "identified" by a software program and mathematically described so that multidimensional parameterization is possible. This refers to obtaining values for 2- and 3-dimensional features, such as measuring the volume of the brain ventricles and compartments, distribution of gene expression and spatiotemporal evolution, as well as co-registration across different samples. To achieve this goal, we have been developing a program named CAFE (Computer Aided Feature Extraction). It originated from an application mainly to quantify filaments in 2D images (Hwang and Eryilmaz, 2014). A distinct feature of CAFE is its synergy between image analysis and model building. Since pixel data are affected by experimental conditions, CAFE initially uses pixel data to build a coarse-grained, ball-and-stick model of the system being imaged (Figure 6). Working on geometric elements instead of pixel data increases calculation speed and allows for mathematical descriptions of the model. For example, for the ball-and-stick model of a zebrafish brain, another model from a different imaging channel (e.g., fluorescence image stacks of gene expression) can be overlaid, which will be useful for analyzing fluorophore spatial relation (Figures 6A-C). Once 
A

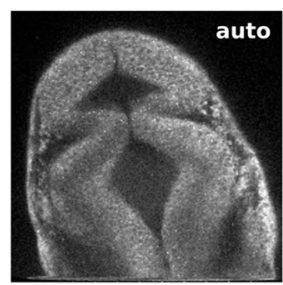

B

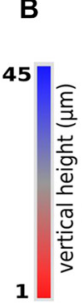

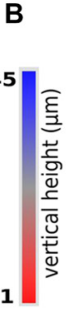

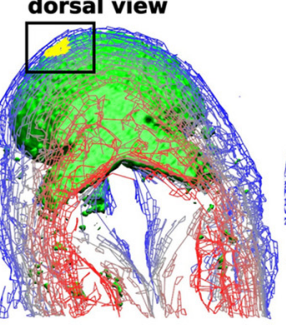

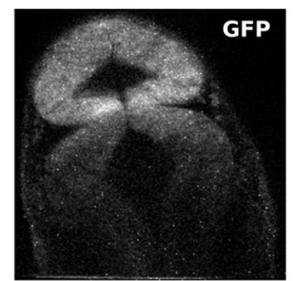

dorsal view
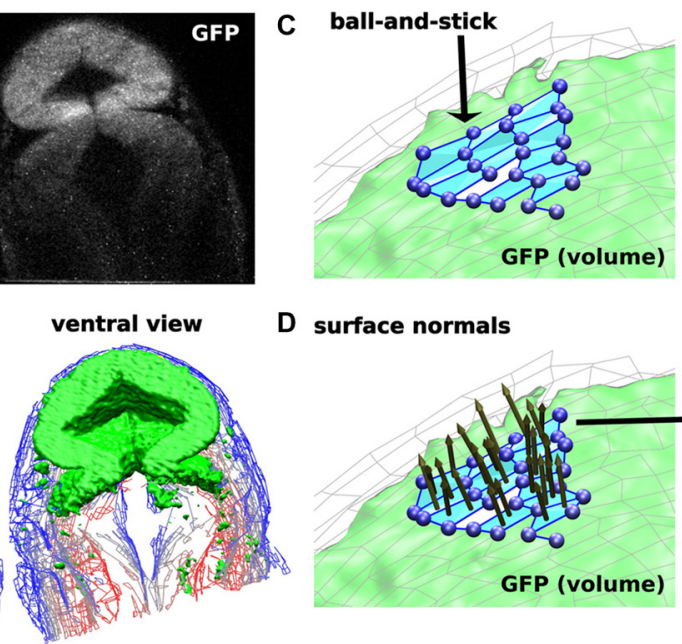

D surface normals

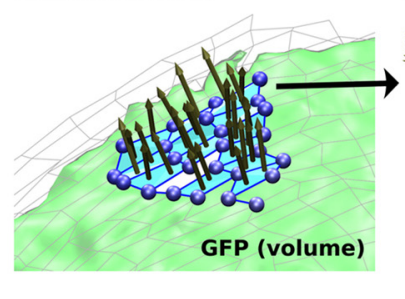

E

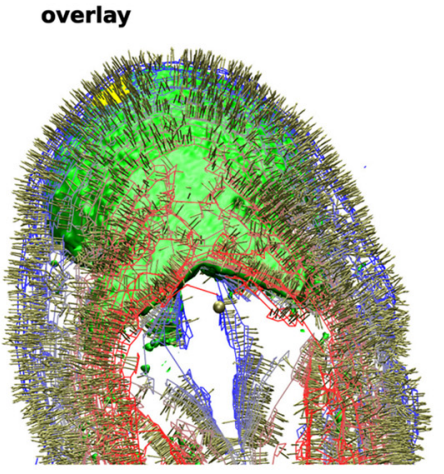

FIGURE 6 | Computer Aided Feature Extraction (CAFE) reconstruction of zebrafish brain embryo images. (A) Single slice 2-photon microscope images of zebrafish brain embryo at 20 hpf displaying autofluorescence (left) and GFP (right) channels. GFP channel marks wnt1 expression. (B) Zebrafish embryo structure representation of autofluorescence (wires) and GFP (green volume). Visualizing images 1-45 in the 79-image stack in dorsal (left) and ventral (right) views. Autofluorescence (wires) reconstructed using boundary-based detection function to recognize local pixel gradients and define ball-and-stick model along the edges of the image. GFP (green volume) reconstructed using an area-based detection function to place balls in areas of high pixel intensity. (C) Detailed ball-and-stick model of boxed region in (B) showing CAFE-defined morphological surface (light blue). (D) Local surface normals for surfaces highlighted in (C). (E) Dorsal view of overlay of autofluorescence and GFP reconstructions with surface normals.

the ball-and-stick model is constructed, additional mathematical operations are possible, such as defining surface normals (Figures 6D,E).

To the goal of obtaining a comprehensive spatio-temporal atlas of embryonic development, a tool such as CAFE is indispensable. Ultimately, quantitative description of the development process will be necessary. For example, the evolution of the volume and morphology of different brain compartments at various time points and distribution of gene reporters across the brain and their time evolution are imperative relationships that would vary in pathological development including neurodevelopmental diseases. Further, in order to build a "canonical" model of zebrafish brain development, measurements described above have to be made across images of many different embryos, and averaged at each time point, which requires co-registration (Gibbs et al., 2014b). Building such canonical models is a major goal of structural bioimage informatics.

Analysis between data samples will quantify the variability of morphogenetic changes and provide a defined basis for conclusions in developmental studies. Current image processing tools vary in terms of compatibility with imaging modality, user interface, and extent of automated analysis. Since the zebrafish is an ideal model organism that can be used to classify both gene expression and morphology in vertebrates, there are several software applications developed exclusively for highcontent, high-throughput zebrafish imaging data. Applications range from cell tracking to generating a $3 \mathrm{D}$ brain atlas of zebrafish larvae, which allow researchers to address questions in developmental biology, neuronal pathways, genetics, etc. (Ronneberger et al., 2012; Mikut et al., 2013; Gibbs et al., 2014b).
An advanced zebrafish-specific application is ViBE-Z, a web-based framework that performs colocalization of zebrafish larvae at 48, 72, and 96 hpf (Ronneberger et al., 2012). ViBE-Z requires manual selection of morphological landmarks from a training set of images to perform sequential registration techniques and align test samples to a training set. Using image colocalization, ViBE-Z can segment anatomical regions of the zebrafish larva and visualize gene expression patterns in the zebrafish volume. The restriction on sample time points that can be analyzed, as well as the needed similarity between samples for colocalization, poses a problem when analyzing early-stage (less than $24 \mathrm{hpf}$ ) zebrafish embryos with minimal major features or mutant phenotypes. In addition, a major limitation of ViBE-Z, and other zebrafish imaging software, is the lacking potential for quantitative measurements. Obtaining volume-based measurements is challenging since multiple images, a reference coordinate set, and definitive markers at a particular location within a coordinate set are needed. As an example, ViBE-Z appropriately co-registers several image samples and visualizes where, relative to anatomical regions, a gene is expressed in the volume, but it does not measure volume of anatomical regions or distribution of gene expression, nor allow for temporal comparisons.

CAFE services the need in image analysis software tools by building a volumetric model from image stacks based on a Cartesian coordinate system. CAFE uses the reconstructed ball-and-stick model based on the original image's local pixel intensities to perform image-processing steps, such as increase intensity or rotate the image, as well as obtain measurements of $2 \mathrm{D}$ and $3 \mathrm{D}$ features. Normals to the surface can be calculated from the surface patches shown in Figure 6C. Figures 6D,E show 
these surface normals. For volumetric analysis, CAFE can detect the boundary surrounding a user-defined open region and define its general geometry. This parameter is the $3 \mathrm{D}$ complement of the midbrain ventricle area parameter derived from 2-D cross sections (Gibbs et al., 2014b). Pixel-based algorithms have been developed and implemented into several imageprocessing platforms to perform image analysis calculations. CAFE's ability to produce $2 \mathrm{D}$ and $3 \mathrm{D}$ ball-and-stick models and use these representations to obtain quantitative calculations will enhance the ability to extract measurements from multiple image samples. Researchers will be able to quantitatively classify spatiotemporal changes in a developing embryo and likewise mark characteristic effects of missing gene reporters, such as in the ace phenotype.

\section{CONCLUSION}

Crucial to understand for its role in brain development and potential contributions to human brain patterning birth defects, the $\mathrm{MHB}$ is also a remarkable model for the dissection of signaling control of cell fate and tissue morphogenesis. In this review, we present evidence that the Wnt-Fgf signaling interface is correlated with specific morphogenetic changes that drive MHB morphogenesis. The complexity of MHB patterning and morphogenesis creates an imperative to apply new methodologies and approaches to uncover its underlying molecular nature. The simultaneous generation of correct brain morphology, cell types, and neural circuitry is a daunting challenge but is a very robust and adaptable process. Cell behaviors such as differential adhesion, growth and apoptosis, migration and cytoskeletal remodeling must be precisely coordinated over large regions within the developing neuroepithelium and this achievement is so astounding that it is understandable we have been both fascinated and frustrated

\section{REFERENCES}

Babb, S. G., Barnett, J., Doedens, A. L., Cobb, N., Liu, Q., Sorkin, B. C., et al. (2001). Zebrafish E-cadherin: expression during early embryogenesis and regulation during brain development. Dev. Dyn. 221, 231-237. doi: 10.1002/dv dy. 1132

Bae, Y. K., Kani, S., Shimizu, T., Tanabe, K., Nojima, H., Kimura, Y., et al. (2009). Anatomy of zebrafish cerebellum and screen for mutations affecting its development. Dev. Biol. 330, 406-426. doi: 10.1016/j.ydbio.2009. 04.013

Buckles, G. R., Thorpe, C. J., Ramel, M. C., and Lekven, A. C. (2004). Combinatorial Wnt control of zebrafish midbrain-hindbrain boundary formation. Mech. Dev. 121, 437-447. doi: 10.1016/s0925-4773(04)00074-7

Carletti, B., and Rossi, F. (2008). Neurogenesis in the cerebellum. Neuroscientist 14, 91-100. doi: 10.1177/1073858407304629

Chapouton, P., Adolf, B., Leucht, C., Tannhäuser, B., Ryu, S., Driever, W., et al. (2006). her5 expression reveals a pool of neural stem cells in the adult zebrafish midbrain. Development 133, 4293-4303. doi: 10.1242/dev.02573

Chen, B. C., Legant, W. R., Wang, K., Shao, L., Milkie, D. E., Davidson, M. W., et al. (2014). Lattice light-sheet microscopy: imaging molecules to embryos at high spatiotemporal resolution. Science 346:1257998. doi: 10.1126/science. 1257998

Chi, C. L., Martinez, S., Wurst, W., and Martin, G. R. (2003). The isthmic organizer signal FGF8 is required for cell survival in the prospective midbrain and cerebellum. Development 130, 2633-2644. doi: 10.1242/dev.00487 with our attempts to understand the process as a whole. Moving forward to a more complete mechanistic understanding connecting the earliest patterning events with eventual brain architecture and cell fates will require enhanced cooperation between disciplines so that the best possible models can be formulated and thoroughly tested. There are still many open questions regarding how correct brain architecture is formed and cell types designated during neural development, though a thorough understanding of these processes is of extreme importance from a basic science perspective and also for advancement of regenerative medicine in neural disease.

\section{AUTHOR CONTRIBUTIONS}

All authors developed and wrote the manuscript.

\section{FUNDING}

The work was supported by National Institutes of Health (Grant R01NS088564). AC-G was funded by the National Science Foundation Graduate Research Fellowship under Grant No. DGE 1252521. Any opinion, findings, and conclusions or recommendations expressed in this material are those of the authors and do not necessarily reflect the views of the National Science Foundation.

\section{ACKNOWLEDGMENTS}

The authors thank David Green, Saurav Mohanty, and Amy Whitener for comments on the manuscript. Some of the information included in this manuscript was previously published in the doctoral dissertation of Gibbs (2014), available online.

Ciani, L., and Salinas, P. C. (2005). WNTs in the vertebrate nervous system: from patterning to neuronal connectivity. Nat. Rev. Neurosci. 6, 351-362. doi: $10.1038 /$ nrn1665

Clark, C. E. J., Nourse, C. C., and Cooper, H. M. (2012). The tangled web of non-canonical wnt signalling in neural migration. Neurosignals 20, 202-220. doi: 10.1159/000332153

Cohen, E. D., Tian, Y., and Morrisey, E. E. (2008). Wnt signaling: an essential regulator of cardiovascular differentiation, morphogenesis and progenitor self-renewal. Development 135, 789-798. doi: 10.1242/dev. 016865

Cooper, S. R., Emond, M. R., Duy, P. Q., Liebau, B. G., Wolman, M. A., and Jontes, J. D. (2015). Protocadherins control the modular assembly of neuronal columns in the zebrafish optic tectum. J. Cell Biol. 211, 807-814. doi: $10.1083 /$ jcb. 201507108

Cox, W. G., and Hemmati-Brivanlou, A. (1995). Caudalization of neural fate by tissue recombination and bFGF. Development 121, 4349-4358.

Dailey, L., Ambrosetti, D., Mansukhani, A., and Basilico, C. (2005). Mechanisms underlying differential responses to FGF signaling. Cytokine Growth Factor Rev. 16, 233-247. doi: 10.1016/j.cytogfr.2005.01.007

Davey, C. F., and Moens, C. B. (2017). Planar cell polarity in moving cells: think globally, act locally. Development 144, 187-200. doi: 10.1242/dev. 122804

de Chaumont, F., Dallongeville, S., Chenouard, N., Herve, N., Pop, S., Provoost, T., et al. (2012). Icy: an open bioimage informatics platform for extended reproducible research. Nat. Methods 9, 690-696. doi: 10.1038/nmeth.2075 
Doherty, D., Millen, K. J., and Barkovich, A. J. (2013). Midbrain and hindbrain malformations: advances in clinical diagnosis, imaging, and genetics. Lancet Neurol. 12, 381-393. doi: 10.1016/s1474-4422(13)70024-3

Dorsky, R. I., Itoh, M., Moon, R. T., and Chitnis, A. (2003). Two tcf3 genes cooperate to pattern the zebrafish brain. Development 130, 1937-1947. doi: $10.1242 /$ dev.00402

Dworkin, S., and Jane, S. M. (2013). Novel mechanisms that pattern and shape the midbrain-hindbrain boundary. Cell. Mol. Life Sci. 70, 3365-3374. doi: $10.1007 / \mathrm{s} 00018-012-1240-\mathrm{x}$

Dyer, C., Blanc, E., Hanisch, A., Roehl, H., Otto, G. W., Yu, T., et al. (2014). A bi-modal function of Wnt signalling directs an FGF activity gradient to spatially regulate neuronal differentiation in the midbrain. Development 141, 63-72. doi: $10.1242 /$ dev. 099507

Eliceiri, K. W., Berthold, M. R., Goldberg, I. G., Ibáñez, L., Manjunath, B. S., Martone, M. E., et al. (2012). Biological imaging software tools. Nat. Methods 9, 697-710. doi: 10.1038/nmeth.2084

Filas, B. A., Oltean, A., Majidi, S., Bayly, P. V., Beebe, D. C., and Taber, L. A. (2012). Regional differences in actomyosin contraction shape the primary vesicles in the embryonic chicken brain. Phys. Biol. 9:066007. doi: 10.1088/14783975/9/6/066007

Foucher, I., Mione, M., Simeone, A., Acampora, D., Bally-Cuif, L., and Houart, C. (2006). Differentiation of cerebellar cell identities in absence of Fgf signalling in zebrafish Otx morphants. Development 133, 1891-1900. doi: 10.1242/dev. 02352

Gao, L., Shao, L., Higgins, C. D., Poulton, J. S., Peifer, M., Davidson, M. W., et al. (2012). Noninvasive imaging beyond the diffraction limit of 3D dynamics in thickly fluorescent specimens. Cell 151, 1370-1385. doi: 10.1016/j.cell. 2012.10.008

Gato, A., and Desmond, M. E. (2009). Why the embryo still matters: CSF and the neuroepithelium as interdependent regulators of embryonic brain growth, morphogenesis and histiogenesis. Dev. Biol. 327, 263-272. doi: 10.1016/j.ydbio. 2008.12.029

Gibbs, H. C. (2014). Digitizing Vertebrate Brain Development with Ultrashort Pulse Microscopy. Texas A\&M University: Dissertation College Station (TX).

Gibbs, H. C., Bai, Y. Q., Lekven, A. C., and Yeh, A. T. (2014a). Imaging embryonic development with ultrashort pulse microscopy. Opt. Eng. 53:051506. doi: 10.1117/1.oe.53.5.051506

Gibbs, H. C., Dodson, C. R., Bai, Y. Q., Lekven, A. C., and Yeh, A. T. (2014b). Combined lineage mapping and gene expression profiling of embryonic brain patterning using ultrashort pulse microscopy and image registration. J. Biomed. Opt. 19:126016. doi: 10.1117/1.jbo.19.12.126016

Gibbs, H. C., Dodson, C. R., Bai, Y., Lekven, A. C., and Yeh, A. T. (2013). "Combined lineage mapping and fate specification profiling with NLOM-OCM using sub-10-fs pulses," in Proceedings SPIE 8593, Optical Methods in Developmental Biology. doi:10.1117/12.2004330

Green, D., Whitener, A. E., Mohanty, S., and Lekven, A. C. (2015). Vertebrate nervous system posteriorization: grading the function of wnt signaling. Dev. Dyn. 244, 507-512. doi: 10.1002/dvdy.24230

Gutzman, J. H., Graeden, E. G., Lowery, L. A., Holley, H. S., and Sive, H. (2008). Formation of the zebrafish midbrain-hindbrain boundary constriction requires laminin-dependent basal constriction. Mech. Dev. 125, 974-983. doi: 10.1016/j. mod.2008.07.004

Gutzman, J. H., Sahu, S. U., and Kwas, C. (2015). Non-muscle myosin IIA and IIB differentially regulate cell shape changes during zebrafish brain morphogenesis. Dev. Biol. 397, 103-115. doi: 10.1016/j.ydbio.2014.10.017

Gutzman, J. H., and Sive, H. (2010). Epithelial relaxation mediated by the myosin phosphatase regulator Mypt1 is required for brain ventricle lumen expansion and hindbrain morphogenesis. Development 137, 795-804. doi: 10.1242/dev. 042705

Harding, M. J., and Nechiporuk, A. V. (2012). Fgfr-Ras-MAPK signaling is required for apical constriction via apical positioning of Rho-associated kinase during mechanosensory organ formation. Development 139, 3130-3135. doi: $10.1242 /$ dev.082271

Hashimoto, M., and Hibi, M. (2012). Development and evolution of cerebellar neural circuits. Dev. Growth Differ. 54, 373-389. doi: 10.1111/j.1440-169x.2012. 01348.x

Heimbucher, T., Murko, C., Bajoghli, B., Aghaallaei, N., Huber, A., Stebegg, R., et al. (2007). Gbx2 and Otx2 interact with the WD40 domain of Groucho/Tle corepressors. Mol. Cell. Biol. 27, 340-351. doi: 10.1128/mcb.00811-06
Heisenberg, C. P., and Bellaïche, Y. (2013). Forces in tissue morphogenesis and patterning. Cell 153, 948-962. doi: 10.1016/j.cell.2013.05.008

Heisenberg, C. P., Tada, M., Rauch, G. J., Saúde, L., Concha, M. L., Geisler, R., et al. (2000). Silberblick/Wnt11 mediates convergent extension movements during zebrafish gastrulation. Nature 405, 76-81. doi: 10.1038/35011068

Heuberger, J., and Birchmeier, W. (2010). Interplay of cadherin-mediated cell adhesion and canonical wnt signaling. Cold Spring Harb. Perspect. Biol. 2:a002915. doi: 10.1101/cshperspect.a002915

Hidalgo-Sánchez, M., Millet, S., Bloch-Gallego, E., and AlvaradoMallart, R. M. (2005). Specification of the meso-isthmo-cerebellar region: the Otx2/Gbx2 boundary. Brain Res. Rev. 49, 134-149. doi: 10.1016/j. brainresrev.2005.01.010

Huisken, J., Swoger, J., Del Bene, F., Wittbrodt, J., and Stelzer, E. H. K. (2004). Optical sectioning deep inside live embryos by selective plane illumination microscopy. Science 305, 1007-1009. doi: 10.1126/science.1100035

Huisken, J. (2012). Slicing embryos gently with laser light sheets. Bioessays 34, 406-411. doi: 10.1002/bies.201100120

Hwang, W., and Eryilmaz, E. (2014). Kinetic signature of fractal-like filament networks formed by orientational linear epitaxy. Phys. Rev. Lett. 113:025502. doi: 10.1103/physrevlett.113.025502

Inoue, F., Kurokawa, D., Takahashi, M., and Aizawa, S. (2012). Gbx2 directly restricts Otx2 expression to forebrain and midbrain, competing with class III POU factors. Mol. Cell. Biol. 32, 2618-2627. doi: 10.1128/MCB. 00083-12

Jászai, J., Reifers, F., Picker, A., Langenberg, T., and Brand, M. (2003). Isthmusto-midbrain transformation in the absence of midbrain-hindbrain organizer activity. Development 130, 6611-6623. doi: 10.1242/dev.00899

Jungbluth, S., Larsen, C., Wizenmann, A., and Lumsden, A. (2001). Cell mixing between the embryonic midbrain and hindbrain. Curr. Biol. 11, 204-207. doi: 10.1016/s0960-9822(01)00049-5

Kasza, K. E., and Zallen, J. A. (2011). Dynamics and regulation of contractile actin-myosin networks in morphogenesis. Curr. Opin. Cell Biol. 23, 30-38. doi: 10.1016/j.ceb.2010.10.014

Keller, P. J., Schmidt, A. D., Santella, A., Khairy, K., Bao, Z. R., Wittbrodt, J., et al. (2010). Fast, high-contrast imaging of animal development with scanned light sheet-based structured-illumination microscopy. Nat. Methods 7, 637-642. doi: $10.1038 /$ nmeth. 1476

Keller, P., Schmidt, A., Wittbrodt, J., and Stelzer, E. (2008). Reconstruction of zebrafish early embryonic development by scanned light sheet microscopy. Science 322, 1065-1069. doi: 10.1126/science.1162493

Keller, P. J., and Stelzer, E. H. K. (2008). Quantitative in vivo imaging of entire embryos with digital scanned laser light sheet fluorescence microscopy. Curr. Opin. Neurobiol. 18, 624-632. doi: 10.1016/j.conb.2009.03.008

Kiecker, C., and Niehrs, C. (2001). A morphogen gradient of Wnt/betacatenin signalling regulates anteroposterior neural patterning in Xenopus. Development 128, 4189-4201.

Kim, S. A., Tai, C. Y., Mok, L. P., Mosser, E. A., and Schuman, E. M. (2011). Calcium-dependent dynamics of cadherin interactions at cell-cell junctions. Proc. Natl. Acad. Sci. U S A 108, 9857-9862. doi: 10.1073/pnas. 1019003108

Kishimoto, N., Alfaro-Cervello, C., Shimizu, K., Asakawa, K., Urasaki, A., Nonaka, S., et al. (2011). Migration of neuronal precursors from the telencephalic ventricular zone into the olfactory bulb in adult zebrafish. J. Comp. Neurol. 519, 3549-3565. doi: 10.1002/cne.22722

Klymkowsky, M. W., Rossi, C. C., and Artinger, K. B. (2010). Mechanisms driving neural crest induction and migration in the zebrafish and Xenopus laevis. Celll Adhes. Migr. 4, 595-608. doi: 10.4161/cam.4.4.12962

Knosp, W. M., Knox, S. M., Lombaert, I. M. A., Haddox, C. L., Patel, V. N., and Hoffman, M. P. (2015). Submandibular parasympathetic gangliogenesis requires sprouty-dependent Wnt signals from epithelial progenitors. Dev. Cell 32, 667-677. doi: 10.1016/j.devcel.2015.01.023

Kurokawa, D., Ohmura, T., Akasaka, K., and Aizawa, S. (2012). A lineage specific enhancer drives Otx2 expression in teleost organizer tissues. Mech. Dev. 128, 653-661. doi: 10.1016/j.mod.2011.11.001

Langenberg, T., and Brand, M. (2005). Lineage restriction maintains a stable organizer cell population at the zebrafish midbrain-hindbrain boundary. Development 132, 3209-3216. doi: 10.1242/dev.01862

Lapebie, P., Borchiellini, C., and Houliston, E. (2011). Dissecting the PCP pathway: one or more pathways? Does a separate Wnt-Fz-Rho 
pathway drive morphogenesis? Bioessays 33, 759-768. doi: 10.1002/bies. 201100023

Lassiter, R. N. T., Stark, M. R., Zhao, T. Y., and Zhou, C. J. J. (2014). Signaling mechanisms controlling cranial placode neurogenesis and delamination. Dev. Biol. 389, 39-49. doi: 10.1016/j.ydbio.2013.11.025

Lekven, A. C., Buckles, G. R., Kostakis, N., and Moon, R. T. (2003). wnt1 and wnt10b function redundantly at the zebrafish midbrain-hindbrain boundary. Dev. Biol. 254, 172-187. doi: 10.1016/s0012-1606(02)00044-1

Lele, Z., Folchert, A., Concha, M., Rauch, G. J., Geisler, R., Rosa, F., et al. (2002). Parachute/n-cadherin is required for morphogenesis and maintained integrity of the zebrafish neural tube. Development 129, 3281-3294.

Lin, M. J., and Lee, S. J. (2016). Stathmin-like 4 is critical for the maintenance of neural progenitor cells in dorsal midbrain of zebrafish larvae. Sci. Rep. 6:36188. doi: $10.1038 /$ srep 36188

Lowery, L. A., De Rienzo, G., Gutzman, J. H., and Sive, H. (2009). Characterization and classification of zebrafish brain morphology mutants. Anat. Rec. (Hoboken) 292, 94-106. doi: 10.1002/ar.20768

Lowery, L. A., and Sive, H. (2004). Strategies of vertebrate neurulation and a re-evaluation of teleost neural tube formation. Mech. Dev. 121, 1189-1197. doi: $10.1016 /$ j.mod.2004.04.022

Mahou, P., Zimmerley, M., Loulier, K., Matho, K. S., Labroille, G., Morin, X., et al. (2012). Multicolor two-photon tissue imaging by wavelength mixing. Nat. Methods 9, 815-818. doi: 10.1038/nmeth.2098

Martinez, S., Crossley, P. H., Cobos, I., Rubenstein, J. L. R., and Martin, G. R. (1999). FGF8 induces formation of an ectopic isthmic organizer and isthmocerebellar development via a repressive effect on Otx2 expression. Development 126, 1189-1200.

McGrew, L. L., Lai, C.-J., and Moon, R. T. (1995). Specification of the anteroposterior neural axis through synergistic interaction of the Wnt signaling cascade with noggin and follistatin. Dev. Biol. 172, 337-342. doi: 10.1006/dbio. 1995.0027

McMahon, A. P., and Bradley, A. (1990). The Wnt-1 (int-1) proto-oncogene is required for development of a large region of the mouse brain. Cell 62, 1073-1085. doi: 10.1016/0092-8674(90)90385-r

McMahon, A., Supatto, W., Fraser, S. E., and Stathopoulos, A. (2008). Dynamic analyses of Drosophila gastrulation provide insights into collective cell migration. Science 322, 1546-1550. doi: 10.1126/science.11 67094

Megason, S. G., and McMahon, A. P. (2002). A mitogen gradient of dorsal midline Wnts organizes growth in the CNS. Development 129, 2087-2098.

Meier, F., Giesert, F., Delic, S., Faus-Kessler, T., Matheus, F., Simeone, A., et al. (2014). FGF/FGFR2 signaling regulates the generation and correct positioning of bergmann glia cells in the developing mouse cerebellum. PLoS One 9:e101124. doi: 10.1371/journal.pone.0101124

Mikut, R., Dickmeis, T., Driever, W., Geurts, P., Hamprecht, F. A., Kausler, B. X., et al. (2013). Automated processing of zebrafish imaging data: a survey. Zebrafish 10, 401-421. doi: 10.1089/zeb.2013.0886

Millen, K. J., Steshina, E. Y., Iskusnykh, I. Y., and Chizhikov, V. V. (2014). Transformation of the cerebellum into more ventral brainstem fates causes cerebellar agenesis in the absence of ptfla function. Proc. Natl. Acad. Sci. U S A 111, E1777-E1786. doi: 10.1073/pnas.1315024111

Miyake, A., and Itoh, N. (2013). Fgf22 regulated by Fgf3/Fgf8 signaling is required for zebrafish midbrain development. Biol. Open 2, 515-524. doi: 10.1242/bio. 20134226

Miyake, A., Nihno, S., Murakoshi, Y., Satsuka, A., Nakayama, Y., and Itoh, N. (2012). Neucrin, a novel secreted antagonist of canonical Wnt signaling, plays roles in developing neural tissues in zebrafish. Mech. Dev. 128, 577-590. doi: 10.1016/j.mod.2012.01.001

Moon, R. T., Kohn, A. D., De Ferrari, G. V., and Kaykas, A. (2004). WNT and beta-catenin signalling: diseases and therapies. Nat. Rev. Genet. 5, 689-701. doi: $10.1038 / \mathrm{nrg} 1427$

Ninkovic, J., Tallafuss, A., Leucht, C., Topczewski, J., Tannhäuser, B., SolnicaKrezel, L., et al. (2005). Inhibition of neurogenesis at the zebrafish midbrain-hindbrain boundary by the combined and dose-dependent activity of a new hairylE(spl) gene pair. Development 132, 75-88. doi: 10.1242/ dev. 01525

Nordström, U., Jessell, T. M., and Edlund, T. (2002). Progressive induction of caudal neural character by graded Wnt signaling. Nat. Neurosci. 5, 525-532. doi: $10.1038 / \mathrm{nn} 854$
Nyholm, M. K., Abdelilah-Seyfried, S., and Grinblat, Y. (2009). A novel genetic mechanism regulates dorsolateral hinge-point formation during zebrafish cranial neurulation. J. Cell Sci. 122, 2137-2148. doi: 10.1242/jcs.043471

Olivier, N., Luengo-Oroz, M. A., Duloquin, L., Faure, E., Savy, T., Veilleux, I., et al. (2010). Cell lineage reconstruction of early zebrafish embryos using label-free nonlinear microscopy. Science 329, 967-971. doi: 10.1126/science.1189428

Ota, S., Taimatsu, K., Yanagi, K., Namiki, T., Ohga, R., Higashijima, S., et al. (2016). Functional visualization and disruption of targeted genes using CRISPR/Cas9-mediated eGFP reporter integration in zebrafiah. Sci. Rep. 6:34991. doi: 10.1038/srep34991

Parada, C., Martin, C., Alonso, M. I., Moro, J. A., Bueno, D., and Gato, A. (2005). Embryonic cerebrospinal fluid collaborates with the isthmic organizer to regulate mesencephalic gene expression. J. Neurosci. Res. 82, 333-345. doi: 10.1002/jnr.20618

Parker, M. O., Brock, A. J., Walton, R. T., and Brennan, C. H. (2013). The role of zebrafish (Danio rerio) in dissecting the genetics and neural circuits of executive function. Front. Neural Circuits 7:13. doi: 10.3389/fncir.2013.00063

Peng, H. C. (2008). Bioimage informatics: a new area of engineering biology. Bioinformatics 24, 1827-1836. doi: 10.1093/bioinformatics/btn346

Picker, A., Brennan, C., Reifers, F., Clarke, J. D. W., Holder, N., and Brand, M. (1999). Requirement for the zebrafish mid-hindbrain boundary in midbrain polarisation, mapping and confinement of the retinotectal projection. Development 126, 2967-2978.

Piloto, S., and Schilling, T. F. (2010). Ovo1 links Wnt signaling with N-cadherin localization during neural crest migration. Development 137, 1981-1990. doi: $10.1242 /$ dev.048439

Puelles, E., Acampora, D., Lacroix, E., Signore, M., Annino, A., Tuorto, F., et al. (2003). Otx dose-dependent integrated control of antero-posterior and dorsoventral patterning of midbrain. Nat. Neurosci. 6, 453-460. doi: 10.1038/nn1037

Raible, F., and Brand, M. (2004). Divide et Impera-the midbrain-hindbrain boundary and its organizer. Trends Neurosci. 27, 727-734. doi: 10.1016/j.tins. 2004.10.003

Rapaciolii, M., Palma, V., and Flores, V. (2016). Morphogenetic and histogenetic roles of the temporal-spatial organization of cell proliferation in the vertebrate corticogenesis as revealed by inter-specific analyses of the optic tectum cortex development. Front. Cell. Neurosci. 10:112. doi: 10.3389/fncel.2016.00112

Recher, G., Jouralet, J., Brombin, A., Heuzé, A., Mugniery, E., Hermel, J. M., et al. (2013). Zebrafish midbrain slow-amplifying progenitors exhibit high levels of transcripts for nucleotide and ribosome biogenesis. Development 140 , 4860-4869. doi: 10.1242/dev.099010

Reifers, F., Böhli, H., Walsh, E. C., Crossley, P. H., Stainier, D. Y. R., and Brand, M. (1998). Fgf8 is mutated in zebrafish acerebellar (ace) mutants and is required for maintenance of midbrain-hindbrain boundary development and somitogenesis. Development 125, 2381-2395.

Reim, G., and Brand, M. (2002). Spiel-ohne-grenzen/pou2 mediates regional competence to respond to Fgf8 during zebrafish early neural development. Development 129, 917-933.

Rhinn, M., and Brand, M. (2001). The midbrain-hindbrain boundary organizer. Curr. Opin. Neurobiol. 11, 34-42. doi: 10.1016/s0959-4388(00)00171-9

Rhinn, M., Lun, K., Ahrendt, R., Geffarth, M., and Brand, M. (2009). Zebrafish gbx1 refines the Midbrain-Hindbrain Boundary border and mediates the Wnt8 posteriorization signal. Neural Dev. 4:12. doi: 10.1186/1749-8104-4-12

Rhinn, M., Lun, K., Amores, A., Yan, Y. L., Postlethwait, J. H., and Brand, M. (2003). Cloning, expression and relationship of zebrafish gbx1 and gbx2 genes to Fgf signaling. Mech. Dev. 120, 919-936. doi: 10.1016/s0925-4773(03)00135-7

Rhinn, M., Lun, K., Luz, M., Werner, M., and Brand, M. (2005). Positioning of the midbrain-hindbrain boundary organizer through global posteriorization of the neuroectoderm mediated by Wnt8 signaling. Development 132, 1261-1272. doi: 10.1242/dev.01685

Ronneberger, O., Liu, K., Rath, M., Ruess, D., Mueller, T., Skibbe, H., et al. (2012). ViBE-Z: a framework for 3D virtual colocalization analysis in zebrafish larval brains. Nat. Methods 9, 735-742. doi: 10.1038/nmeth.2076

Sahu, S. U., Visetsouk, M. R., Garde, R. J., Hennes, L., Kwas, C., and Gutzman, J. H. (2017). Calcium signals drive cell shape changes during zebrafish midbrainhindbrain boundary formation. Mol. Biol. Cell 28, 875-882. doi: 10.1091/mbc. E16-08-0561

Sai, X., and Ladher, R. K. (2008). FGF signaling regulates cytoskeletal remodeling during epithelial morphogenesis. Curr. Biol. 18, 976-981. doi: 10.1016/j.cub. 2008.05.049 
Sander, J. D., and Joung, J. K. (2014). CRISPR-Cas systems for editing, regulating and targeting genomes. Nat. Biotechnol. 32, 347-355. doi: 10.1038/nbt. 2842

Santi, P. A. (2011). Light sheet fluorescence microscopy: a review. J. Histochem. Cytochem. 59, 129-138. doi: 10.1369/0022155410394857

Schindelin, J., Rueden, C. T., Hiner, M. C., and Eliceiri, K. W. (2015). The ImageJ ecosystem: an open platform for biomedical image analysis. Mol. Reprod. Dev. 82, 518-529. doi: 10.1002/mrd.22489

Schlessinger, J. (2000). Cell signaling by receptor tyrosine kinases. Cell 103, 211-225. doi: 10.1016/s0092-8674(00)00114-8

Schmitz, B., Papan, C., and Campos-Ortega, J. A. (1993). Neurulation in the anterior trunk region of the zebrafish brachydanio-rerio. Rouxs Arch. Dev. Biol. 202, 250-259. doi: 10.1007/BF00363214

Scholpp, S., and Brand, M. (2004). Endocytosis controls spreading and effective signaling range of Fgf8 protein. Curr. Biol. 14, 1834-1841. doi: 10.1016/j.cub. 2004.09.084

Severino, M., Allegri, A. E. M., Pistorio, A., Roviglione, B., Di Iorgi, N., Maghnie, M., et al. (2014). Midbrain-hindbrain involvement in septo-optic dysplasia. Am. J. Neuroradiol. 35, 1586-1592. doi: 10.3174/ajnr.A3959

Smutny, M., Ákos, Z., Silvia, G., Shamipour, S., Ruprecht, V., Čapek, D., et al. (2017). Friction forces position the neural anlage. Nat. Cell Biol. 19, 306-317. doi: $10.1038 /$ ncb3492

Stanganello, E., Hagemann, A. I. H., Mattes, B., Sinner, C., Meyen, D., Weber, S., et al. (2015). Filopodia-based Wnt transport during vertebrate tissue patterning. Nat. Commun. 6:5846. doi: 10.1038/ncomms6846

Su, C.-Y., Kemp, H. A., and Moens, C. B. (2014). Cerebellar development in the absence of Gbx function in zebrafish. Dev. Biol. 386, 181-190. doi: 10.1016/j. ydbio.2013.10.026

Sunmonu, N. A., Li, K. R., Guo, Q. X., and Li, J. Y. H. (2011). Gbx2 and Fgf8 are sequentially required for formation of the midbrain-hindbrain compartment boundary. Development 138, 725-734. doi: 10.1242/dev.055665

Tallafuss, A., and Bally-Cuif, L. (2003). Tracing of her5 progeny in zebrafish transgenics reveals the dynamics of midbrain-hindbrain neurogenesis and maintenance. Development 130, 4307-4323. doi: 10.1242/dev.00662

Thomas, K. R., and Capecchi, M. R. (1990). Targeted disruption of the murine int-1 proto-oncogene resulting in severe abnormalities in midbrain and cerebellar development. Nature 346, 847-850. doi: 10.1038/346847a0

Tossell, K., Andreae, L. C., Cudmore, C., Lang, E., Muthukrishnan, U., Lumsden, A., et al. (2011a). Lrrn1 is required for formation of the midbrainhindbrain boundary and organiser through regulation of affinity differences between midbrain and hindbrain cells in chick. Dev. Biol. 352, 341-352. doi: 10.1016/j.ydbio.2011.02.002

Tossell, K., Kiecker, C., Wizenmann, A., Lang, E., and Irving, C. (2011b). Notch signalling stabilises boundary formation at the midbrain-hindbrain organiser. Development 138, 3745-3757. doi: 10.1242/dev.070318

Tsai, F. C., Kuo, G. H., Chang, S. W., and Tsai, P. J. (2015). $\mathrm{Ca}^{2+}$ signaling in cytoskeletal reorganization, cell migration, and cancer metastasis. Biomed Res. Int. 2015:409245. doi: 10.1155/2015/409245
Tuazon, F. B., and Mullins, M. C. (2015). Temporally coordinated signals progressively pattern the anteroposterior and dorsoventral body axes. Semin. Cell Dev. Biol. 42, 118-133. doi: 10.1016/j.semcdb.2015.06.003

Valm, A., Cohen, S., Legant, W., Melunis, J., Hershberg, U., Wait, E., et al. (2017). Applying systems-level spectral imaging and analysis to reveal the organelle interactome. Nature 546, 162-167. doi: 10.1038/nature22369

Varelas, X., Bouchie, M. P., and Kukuruzinska, M. A. (2014). Protein $\mathrm{N}$-glycosylation in oral cancer: dysregulated cellular networks among DPAGT1, E-cadherin adhesion and canonical Wnt signaling. Glycobiology 24, 579-591. doi: 10.1093/glycob/cwu031

Volkmann, K., Chen, Y. Y., Harris, M. P., Wullimann, M. F., and Köster, R. W. (2010). The zebrafish cerebellar upper rhombic lip generates tegmental hindbrain nuclei by long-distance migration in an evolutionary conserved manner. J. Comp. Neurol. 518, 2794-2817. doi: 10.1002/cne.22364

Weber, M., and Huisken, J. (2011). Light sheet microscopy for real-time developmental biology. Curr. Opin. Genet. Dev. 21, 566-572. doi: 10.1016/j.gde. 2011.09.009

Woo, K., and Fraser, S. E. (1995). Order and coherence in the fate map of the zebrafish nervous-system. Development 121, 2595-2609.

Wurst, W., and Bally-Cuif, L. (2001). Neural plate patterning: upstream and downstream of the isthmic organizer. Nat. Rev. Neurosci. 2, 99-108. doi: $10.1038 / 35053516$

Yook, J. I., Li, X. Y., Ota, I., Fearon, E. R., and Weiss, S. J. (2005). Wnt-dependent regulation of the E-cadherin repressor snail. J. Biol. Chem. 280, 11740-11748. doi: $10.1074 /$ jbc.m413878200

Yoshiba, S., and Hamada, H. (2014). Roles of cilia, fluid flow, and $\mathrm{Ca}^{2+}$ signaling in breaking of left-right symmetry. Trends Genet. 30, 10-17. doi: 10.1016/j.tig. 2013.09.001

Yu, S. R., Burkhardt, M., Nowak, M., Ries, J., Petrásek, Z., Scholpp, S., et al. (2009). Fgf8 morphogen gradient forms by a source-sink mechanism with freely diffusing molecules. Nature 461, 533-536. doi: 10.1038/nature 08391

Zervas, M., Millet, S., Ahn, S., and Joyner, A. L. (2004). Cell behaviors and genetic lineages of the mesencephalon and rhombomere 1. Neuron 43, 345-357. doi: 10.1016/j.neuron.2004.07.010

Conflict of Interest Statement: The authors declare that the research was conducted in the absence of any commercial or financial relationships that could be construed as a potential conflict of interest.

Copyright (C) 2017 Gibbs, Chang-Gonzalez, Hwang, Yeh and Lekven. This is an open-access article distributed under the terms of the Creative Commons Attribution License (CC BY). The use, distribution or reproduction in other forums is permitted, provided the original author(s) or licensor are credited and that the original publication in this journal is cited, in accordance with accepted academic practice. No use, distribution or reproduction is permitted which does not comply with these terms. 